\title{
Detailed Analysis of the Flow Within the Boundary Layer and Wake of a Full-Scale Ship
}

\author{
Blanca Pena, Ema Muk-Pavic, Patrick Fitzsimmons \\ Department of Mechanical Engineering, University College London, London WC1E 7JE, UK \\ Abstract: This article presents a detailed numerical flow assessment of the boundary layer and wake \\ of a full-scale cargo ship. The assessment was conducted using a sophisticated numerical approach \\ that is able to resolve large turbulent scale vortices contained in the flow. The physical flow features \\ of the boundary layer and wake investigated include mean-velocity, near-wall shear stress and \\ vorticity fields. Also, the evolution of the wake from the thick boundary layer over the stern is \\ displayed and analysed in the highest possible detail. Additionally, the detailed information extracted \\ from the boundary layer and wake was the primary input to assess the overall hydrodynamic \\ efficiency of the full-scale general cargo ship.
}

The analysis method followed during this work has been a determinant factor for fast and efficient design of energy saving devices, propellers or rudders that work within the limits of the boundary layer of a ship. In particular, this thorough analysis avoided the necessity to use the commonly used practice of trial and error that is typically followed in the maritime industry.

Keywords: hydrodynamics, turbulent boundary layer, CFD, full scale, ship efficiency.

\section{Introduction}

Experimental fluid dynamics towing tank tests have been traditionally used to evaluate the flow around the ship. The main principle of this technique is to test a scaled model of the ship in similar conditions to that of a full scale one. This approach is expensive, time-consuming and most importantly carries significant limitations. Among them, due to Reynolds number differences, the fullscale boundary layer is generally thinner than in model scale. Also, the aft boundary layer is significantly different in model and full-scale. For applications such as ship resistance investigations, model to full-scale scaling limitations are usually overcome by the application of empirical correlation factors (Larsson and Raven, 2010). This is not the case for boundary layer investigations where a model to full-scale correlation approach is not yet fully established (ITTC, 2014, ITTC, 2017).

An example of model scale assessments of the boundary layer and wake was conducted by Patel et al. (1990). This type of investigation has been beneficial to a better understanding of the flow behaviour 
on the ship aft end. Nevertheless, when an accurate picture of the full-scale aft end flow is required, a model scale investigation is not the most suitable option.

An alternative method to assess the near-wall flow of the ship relies on viscous flow Computational Fluid Dynamics (CFD). It is based on the Navier-Stokes equations and allows numerical modelling of scenarios in full-scale, therefore avoiding scaling issues. For full-scale ship hydrodynamic applications, Reynolds Averaged Navier-Stokes (RANS) is known to provide a quick solution as it does not require significant computational power. Full-scale self-propulsion studies revealed that RANS numerical model is able to provide good quality predictions of the propeller forces and moment (Ponkratov and Zegos, 2015; Jasak et al., 2019; Bakica et al., 2020). These investigations are in line with the results of the Lloyds Register First Full-scale Ship Hydrodynamics Workshop (Lloyds Register, 2016). However, RANS might not be recommended for scenarios when the flow is predominantly unsteady and/or hull flow separation is expected, this is particularly important for calculations of the bilge vortex that typically forms at the stern of high block coefficient ships (ITTC, 2014).

If flow separation is expected, one could consider the implementation of Large Eddy Simulation (LES) to model the turbulence contained in the flow. The principle of LES is to approach the modelling of turbulence by considering that the large vortical structures created by the geometry contain most of the energy within the bulk flow. LES resolves turbulent vortices everywhere in the flow domain down to the grid size. LES could provide more accurate predictions of the fluid flow than RANS; however, LES is still computationally unaffordable in full-scale ship hydrodynamics due to the high Reynolds number.

A most recent approach for the simulation of turbulent ship flows is based on a combination of RANS/LES, such as DES (Detached Eddy Simulation). This method combines the best features of LES and RANS by only using LES away from the wall where a high level of unsteadiness of the flow is expected (i.e. around the bilges, detached flow regions or in the wake) while RANS is applied in the near-wall region. This method is more computationally affordable in ship hydrodynamics than LES, allowing the study of complex unsteady flows in full-scale and being deemed as the best alternative to calculate wake parameters, especially behind high block coefficient ships (Larsson et al., 2015). Fullscale flow predictions using a DES97 and its improved version, a DDES, were conducted by Xing et al. (2010). The authors established that both DES approaches improve the prediction of the total resistance and velocity distribution for most of the propeller plane; however, the authors also revealed that both models showed issues predicting the shear stress in the boundary layer region. Some of these issues might be attributed to the log-layer mismatch behaviour that the DES97 and DDES models exhibit (Spalart et al., 2006) which could be corrected using an IDDES approach and which represents an improved version of the DDES and DES97 approaches (Shur et al., 2008a). 
This paper presents a thorough analysis of the aft end boundary layer and wake of a full-scale ship. The numerical approach used during this analysis is based on an IDDES approach that was previously validated against sea trials torque data (Pena et al., 2020). Also, the computational mesh was tailored to allow for the resolution of the largest turbulent vortex that is expected to be shed from the hull: the bilge vortex. Nominal wake, resistance distribution and velocity fields have been post-processed to assess the hydrodynamic performance of the 'MV Regal' (Lloyds Register, 2016), a full-scale general cargo ship.

\section{Benchmark Case Study}

The 'Regal' is a $138 \mathrm{~m}$ single screw vessel (Figure 1) with the following main particulars (Table 1):

Table 1 Regal main particulars (Lloyds Register, 2016)

\begin{tabular}{ll}
\hline Parameter & \\
\hline Length between perpendiculars, Lpp & $138 \mathrm{~m}$ \\
Breadth moulded, B & $23 \mathrm{~m}$ \\
Depth moulded, D & $12.1 \mathrm{~m}$ \\
Draught, T & Ballast \\
Propeller diameter, D & $5.2 \mathrm{~m}$ (four-bladed) \\
\hline
\end{tabular}

Before the sea trials, the vessel was dry-docked, the hull was cleaned, and the propeller surface was polished. In this clean condition, the hull, rudder and propeller were 3D laser scanned to obtain an accurate geometric representation. The scanned geometry was directly imported into the CFD computations, thus ensuring high accuracy of the geometry CAD models.

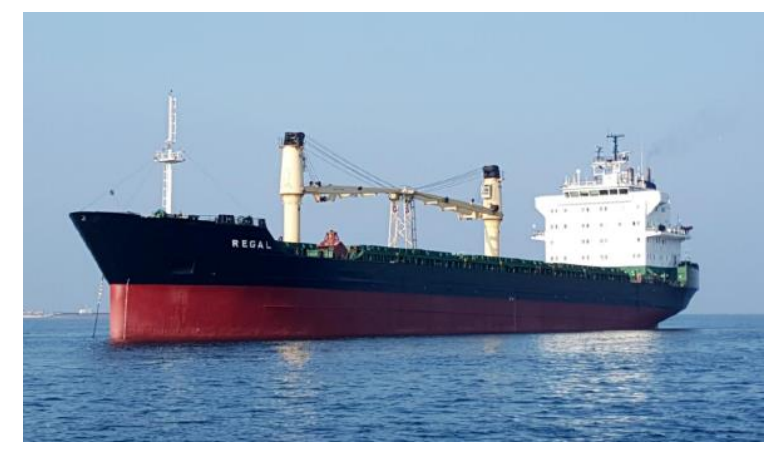

Figure 1 Regal general cargo ship (Lloyds Register, 2016) 
The sea trials were conducted in a reasonably calm condition, in compliance with the ISO 15016:2015 standard (ISO, 2015) and recommended sea trails procedure ITTC 7.5-04-01-01.1 (ITTC, 2014b). The speed trials were conducted at ballast draught at three different shaft speeds.

Therefore, the scope of the analysis corresponds to the programme conducted during the Lloyds Register full-scale Hydrodynamics workshop. These numerical study simulations of the flow around the ship were conducted using the commercial CFD code Siemens Star CCM+. The numerical experiments were undertaken for the full-scale ship Regal at the ballast draft and in a clean hull condition for the bare hull simulations (with rudder only) at the range of speeds given in Table 2 . This set of simulations significantly reduces the complexity of the aft end flow (compared with selfpropulsion tests), allowing to study the nominal wake fields.

Table 2 Bare-hull simulation conditions

\begin{tabular}{lll}
\hline Speed (knots) & Re & Fr \\
\hline 8 & $6.43 \mathrm{E}+08$ & 0.11 \\
10 & $8.03 \mathrm{E}+08$ & 0.14 \\
12 & $9.64 \mathrm{E}+08$ & 0.17 \\
14 & $1.12 \mathrm{E}+09$ & 0.20 \\
\hline
\end{tabular}

\section{Numerical Approach}

\subsection{Turbulence Modelling Strategy}

This work uses the Improved Delayed Detached Eddy Simulation (IDDES) turbulence modelling strategy and following the approach described in previous work (Pena et al., 2019; Pena et al., 2020). The IDDES belongs to the DES family, and it is based on the model developed by Shur et al. (2008). In general, this model switches between the RANS SST k- $\omega$ model, which has demonstrated maturity and reliability calculating skin friction coefficients and steady flow features (Wilcox, 1993; Menter, 1994); and LES in away from the wall, where it can capture the larger unsteady eddies such as the bilge vortex. This approach ensures that attached regions are modelled by RANS whereas the immediate region in front of the propeller (which contains the unsteady bilge vortex) is solved by LES; ensuring that the aftermost region turbulence is better predicted than by using pure RANS.

The IDDES approach is obtained by modifying the dissipation term of the transport equation for the turbulent kinetic energy $(k)$. After introducing a length scale, $L_{h y b r i d}$, the turbulence model equations in tensor form are given as (Shur et al, 2008).: 


$$
\begin{gathered}
\frac{\partial(\rho k)}{\partial t}+\frac{\partial\left(u_{j} k\right)}{\partial x_{j}}=\frac{\partial}{\partial x_{j}}\left[\left(\mu_{l}+\sigma_{k} \mu_{t}\right) \frac{\partial k}{\partial x_{j}}\right]+\tau_{i j} S_{i j}-\frac{\rho k^{3 / 2}}{L_{h y b r i d}} \\
\frac{\partial(\rho \omega)}{\partial t}+\frac{\partial\left(\rho u_{j} \omega\right)}{\partial x_{j}}=\frac{\partial}{\partial x_{j}}\left[\left(\mu_{l}+\sigma_{\omega} \mu_{t}\right) \frac{\partial \omega}{\partial x_{j}}\right]+\alpha \frac{\omega}{k} \tau_{i j} S_{i j}-\frac{\rho k^{3 / 2}}{L_{h y b r i d}}-\beta \rho \omega^{2}+2\left(1-F_{1}\right) \frac{\rho \sigma_{\omega 2}}{\omega} \frac{\partial k}{\partial x_{j}} \frac{\partial \omega}{\partial x_{j}}
\end{gathered}
$$

where $S_{i j}$ represents the strain tensor, $\tau_{i j}$ the stress tensor, $F_{1}$ is the blending function. The length scale, $L_{h y b r i d}$ is defined as:

$$
L_{\text {hybrid }}=f_{B}\left(1+f_{e}\right) L_{R A N S}+\left(1-f_{B}\right) L_{L E S}
$$

where $L_{R A N S}=k^{\frac{1}{2}} /\left(\beta^{*} \omega\right), \beta^{*}$ is given in k-Omega Model Coefficients taken as 0.09. $L_{L E S}=C_{D E S} \Delta$, being $C_{D E S}=0.78$, and $\Delta$ is the grid length scale. The elevating-function $f_{e}$ prevents an excessive reduction of the RANS Reynolds Stresses (Shur et al., 2008b). The key of this model is the empirical blending-function, $f_{B}$, which presents a switching function from RANS $\left(f_{B}=1\right)$ to LES model $\left(f_{B}=\right.$ $0)$.

\subsection{Numerical Model of the Ship}

The ship model was placed in a prismatic fluid domain with the inlet boundary placed one length upstream of the ship's bow, the outlet boundary two ship lengths downstream of the transom of the ship and the sides of the fluid domain one ship length towards the port and the starboard sides as shown in Figure 6 (left). This approach follows the ITTC recommended practices (ITTC, 2014c, Lloyds Register, 2016). A Dirichlet condition was imposed on the inlet. The DFBI (Dynamic Fluid Body Interaction) module was used to simulate the motion of the ship in response to pressure and shear forces exerted by the fluid on the solid body, as well as gravity. The ship model is allowed to sink and trim freely. The resultant force and moment acting on the ship are calculated, and the governing equations of rigid body motion are solved every time step to find the new position of the ship. This approach was selected as it allows for direct comparison of the CFD computation integral forces with benchmark data from the 2016 Workshop in Ship Hydrodynamics (Lloyds Register, 2016).

The properties of the ship that are defined in the simulation (Table 3) correspond to the values determined during the sea trials (Lloyds Register, 2016). 
Table 3 CFD simulation ship parameters

\begin{tabular}{ll}
\hline Parameter & Units \\
\hline Longitudinal Centre of Gravity, LCG & $\begin{array}{l}71.2 \quad \mathrm{~m} \mathrm{(from} \mathrm{aft} \\
\text { perpendicular) }\end{array}$ \\
Vertical Centre of Gravity, VCG & $0 \mathrm{~m}$ (free-surface) \\
Transverse Centre of Gravity, TCG & $0 \mathrm{~m}$ \\
Displacement, $\Delta$ & 12881 tonnes \\
Pitch Radius of Gyration, Ryy & $0.25 \mathrm{~L} \mathrm{~m}$ \\
\hline
\end{tabular}

\section{Boundary Layer Analysis Approach}

As mentioned in the introduction section, this analysis required a higher definition of the boundary layer of the ship that is required to measure the velocity profiles across the 3D ship boundary layer of the. The boundary layer thickness has been defined as the normal wall distance, $\delta$, at which the velocity $\overline{\vec{u}}$ is equal to $0.99 \overline{U_{\infty}}$. Boundary layer parameters are calculated on spanwise cross-section planes at different 'frames' along the ship length $\left(\mathrm{FR}_{\mathrm{i}}\right)$. All planes are parallel to each other, and their normal component is parallel to the ship length vector. Transversal waterline $\left(\mathrm{WL}_{\mathrm{i}}\right)$ and length planes $\left(\mathrm{LG}_{\mathrm{i}}\right)$ are also defined for reference purposes. FR, WL, and LG planes are shown in Figure 2 and Figure 3. Note that FR $1 / 2$ corresponds to the propeller plane, where nominal wake velocity measurements are taken. By contrast, FR10 corresponds to the end of the ship parallel body.

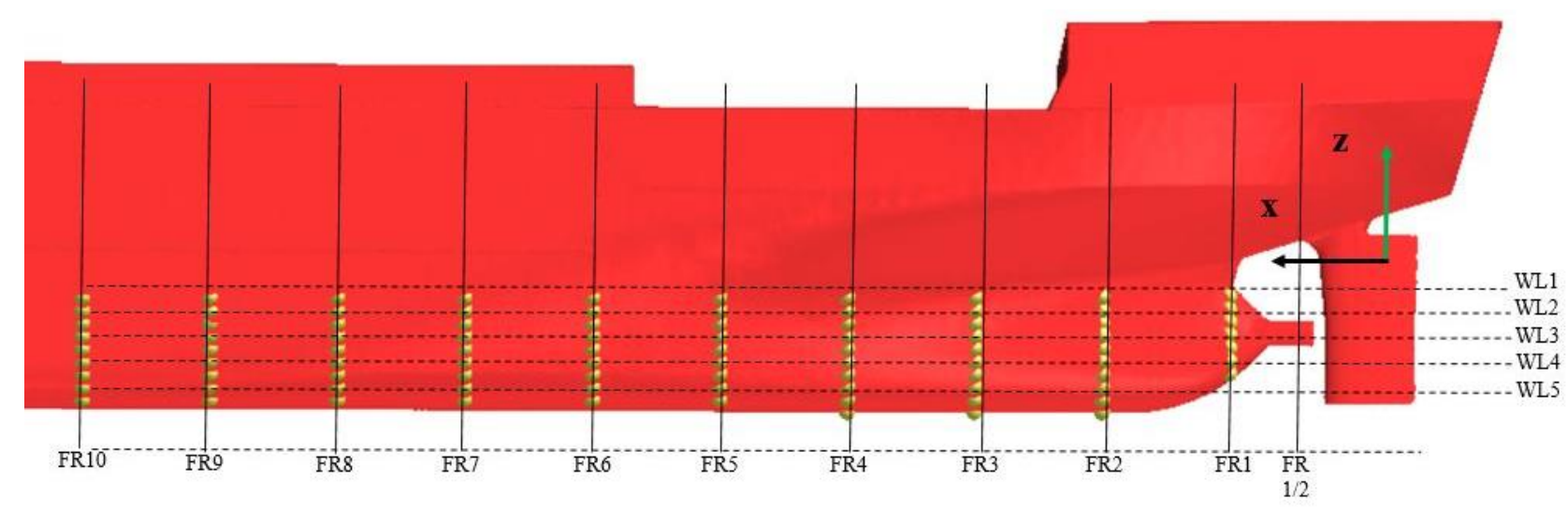

Figure 2 Reference planes for boundary layer numerical measurements 


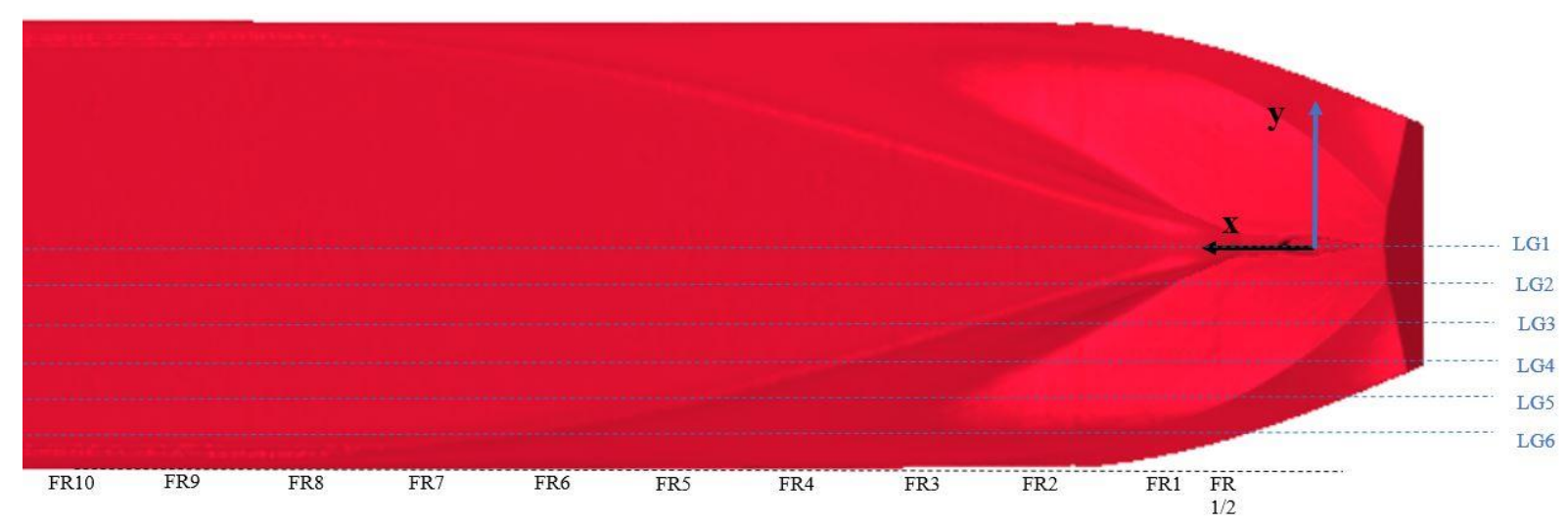

Figure 3 Reference planes for boundary layer numerical measurements

The cartesian coordinates of $\mathrm{FR}_{i}, \mathrm{WL}_{\mathrm{i}}$ and $\mathrm{LG}_{\mathrm{i}}$ planes are given below and are referenced to a righthand cartesian coordinate system with the origin at the intersection of the aft perpendicular and the waterplane. Positive directions of axes are from bow to stern, centre-plane towards the port-side and from the free-surface upwards.

Table 4 Reference planes

\begin{tabular}{|c|c|c|c|c|c|c|c|c|}
\hline $\begin{array}{l}\text { Measurement } \\
\text { Plane }\end{array}$ & $\mathrm{x}_{\mathrm{i}}(\mathrm{m})$ & $\mathrm{x}_{\mathrm{i}} / \mathrm{L}$ & $\begin{array}{l}\text { Measurement } \\
\text { Plane }\end{array}$ & $\mathrm{z}_{\mathrm{i}}(\mathrm{m})$ & $\mathrm{z}_{\mathrm{i}} / \mathrm{T}$ & $\begin{array}{l}\text { Measurement } \\
\text { Plane }\end{array}$ & $\mathrm{y}_{\mathrm{i}}(\mathrm{m})$ & $\mathrm{y} / \mathrm{B}$ \\
\hline FR1/2 & 2.5 & 0.02 & WL1 & -1 & $\begin{array}{l}-0.18 \\
\end{array}$ & LG1 & 0 & 0.00 \\
\hline FR1 & 5 & 0.04 & WL2 & -2 & -0.36 & LG2 & -2 & -0.09 \\
\hline FR2 & 10 & 0.07 & WL3 & -3 & -0.54 & LG3 & -4 & -0.17 \\
\hline FR3 & 15 & 0.11 & WL4 & -4 & -0.72 & LG4 & -6 & -0.26 \\
\hline FR4 & 20 & 0.14 & WL5 & -5 & -0.90 & LG5 & -8 & -0.35 \\
\hline FR5 & 25 & 0.18 & - & - & - & LG6 & - & - \\
\hline FR6 & 30 & 0.22 & - & - & - & - & - & - \\
\hline FR7 & 35 & 0.25 & - & - & - & - & - & - \\
\hline FR8 & 40 & 0.29 & - & - & - & - & - & - \\
\hline FR9 & 45 & 0.33 & - & - & - & - & - & - \\
\hline FR10 & 50 & 0.36 & - & - & - & - & - & - \\
\hline
\end{tabular}

Several probe points are placed during the course of the numerical experiments, as shown in Figure 4. These points are created at the intersection of $\mathrm{FR}_{n}$ and $\mathrm{WL}_{n}$ planes with the hull surface. Each point is therefore identified by an alphanumeric combination based on a FR number and a WL number. For example, the closest point to the free-surface on the FR10 is named PFR10WL1, P (point) on Frame 10 with the intersection of Waterline 1. 
Additional probe points are installed at the flat bottom which are built through the intersection of $\mathrm{LG}_{\mathrm{i}}$ planes, $\mathrm{FR}_{\mathrm{i}}$ planes and the hull surface. The flat bottom probe points on the hull surface are consequently named by an alphanumeric combination of a FR plane followed by a LG longitudinal plane. For example, PFR10LG1 corresponds to the probe point on the intersection of FR10, LG1 and the hull.

An overview of the probe points is shown in Figure 4.

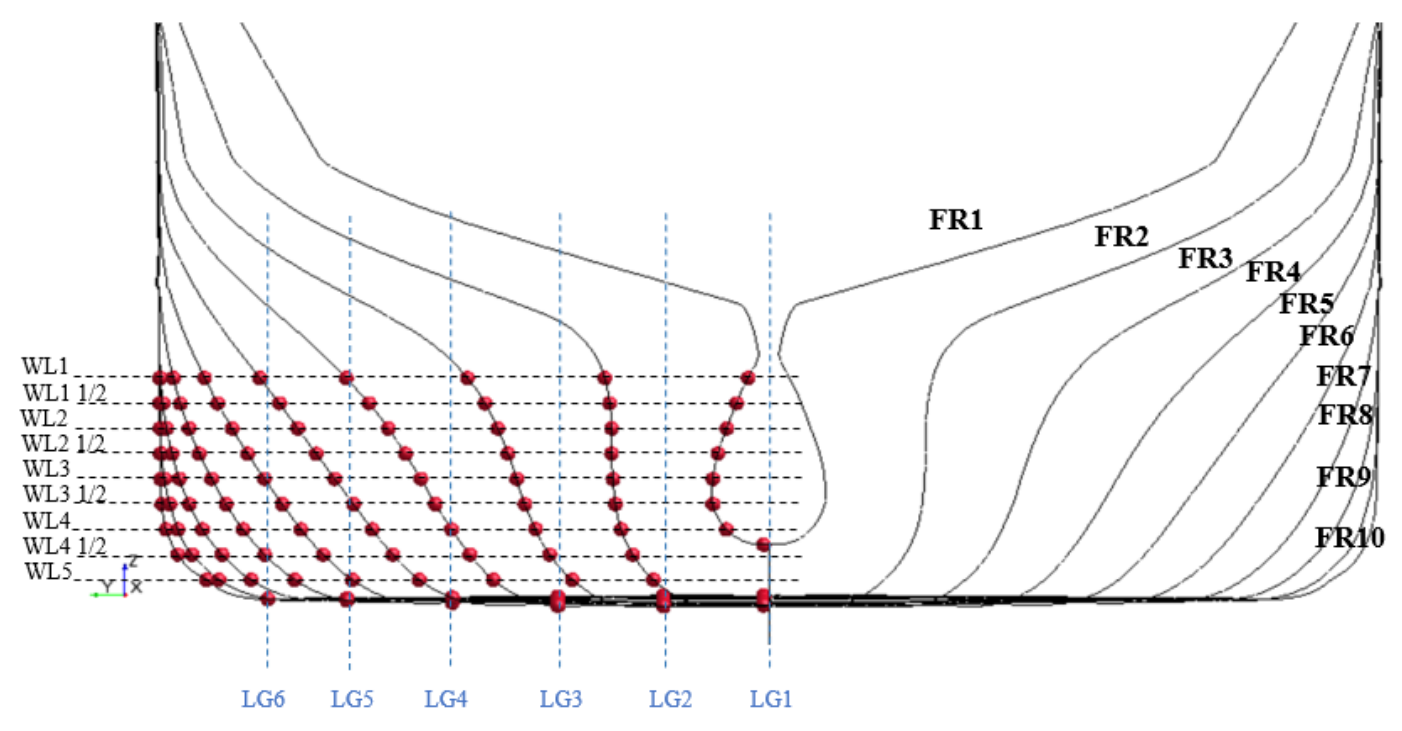

Figure 4 Probe points for boundary layer measurements over the ship hull

Boundary layer mean velocity fields were plotted on each point as $u_{+}=f\left(y_{+}\right)$. The parameters $y_{+}$ and $u_{+}$are defined as: $y_{+}=\frac{\delta u_{\tau}}{v}$ and $u_{+}=\frac{\overline{\vec{u}}}{u_{\tau}}$, where $\overline{\vec{u}}$ is the averaged velocity and $u_{\tau}$ denotes the shear velocity.

\section{Ship Resistance: Calculated Components}

The bare hull total resistance, viscous resistance and pressure resistance are determined from the converged IDDES simulations. The drag forces are made dimensionless and represented by the total resistance coefficient $\left(C_{T}\right)$, pressure resistance coefficient $\left(C_{P}\right)$ and the viscous resistance coefficient $\left(C_{V}\right)$. These coefficients are defined as $C_{T}=\frac{R_{T}}{\frac{1}{2} \rho S \overline{U_{\infty}^{2}}}, C_{V}=\frac{R_{V}}{\frac{1}{2} \rho S \overline{U_{\infty}^{2}}}$ and $C_{P}=\frac{R_{P}}{\frac{1}{2} \rho S \overline{U_{\infty}^{2}}}$, where $R_{T}, R_{v}$ and $R_{P}$ are the total resistance, viscous resistance and pressure resistance, respectively, $\mathrm{S}$ represents the ship wetted surface. Note that $C_{V}$ is calculated using the shear stress tensor and accounts for viscous stresses that the fluid exerts to the hull. 
Ship resistance distribution per unit length is also measured to quantify the drag contribution of each hull segment (i.e. bow, stern). The ship length is divided into fourteen equal intervals of length, as shown in Figure 5. Resistance per unit length (strip) was plotted, where strip 1 is at the Aft Perpendicular and strip 14 is at the Forward Perpendicular (F.P).

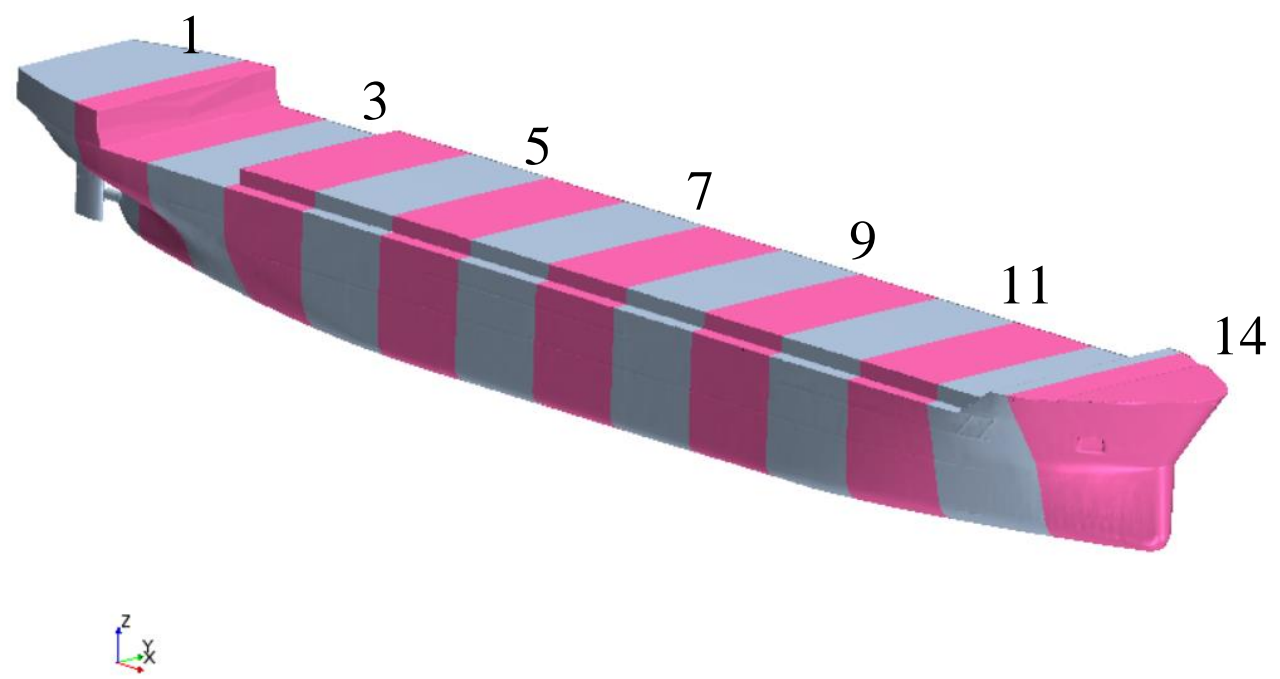

Figure 5 Ship division per unit length

\section{Computational Set-up}

\subsection{Time-step and Spatial Discretization}

The Courant number $\left(\mathrm{C}_{\mathrm{r}}\right)$ is used to represent the number of cells that the fluid travels through within a time step, and it is defined as $\mathrm{C}_{\mathrm{r}}=\frac{u \Delta t}{\Delta x}$ (where $u$ is the local speed, $\Delta t$ the interval of time (time step) and $\Delta x$ the cell size in the direction of the flow). A mean $\mathrm{C}_{\mathrm{r}}$ of 1 is implemented, ensuring the convergence and accuracy of the unsteady simulations. All simulations used a $2^{\text {nd }}$ order spatial and temporal discretisation for all equations.

\subsection{Mesh}

The mesh configuration is identical to previous simulations that were validated against the torque that was measured during the self-propulsion test (Pena et al., 2020) and which consist on a hexahedral structured mesh type was used to discretise the fluid domain with local refinements at the regions of particular interest and where a more detailed resolution of the flow was required. The refinement was made within the wedge region around the ship (Figure 7 left) and the bow to capture flow around the hull and wave pattern (Figure 7 left). A very high-density mesh was generally defined in regions around the stern of the vessel (Figure 6 right), including the rudder. For the free-surface, the volume of fluid method (VOF) was used to capture the deformation of the free-surface. Besides, a second- 
order spatial resolution scheme was used to discretise the free-surface, with a trimmed mesh aligned with the calm water free-surface. With regards to the near-wall cell, the grid was set-up to achieve a mean $y_{+}$of 1 and ensuring that no wall functions are applied in the near-wall region. The final $y_{+}$ configuration is determined during the mesh convergence study, as presented in Table 5.
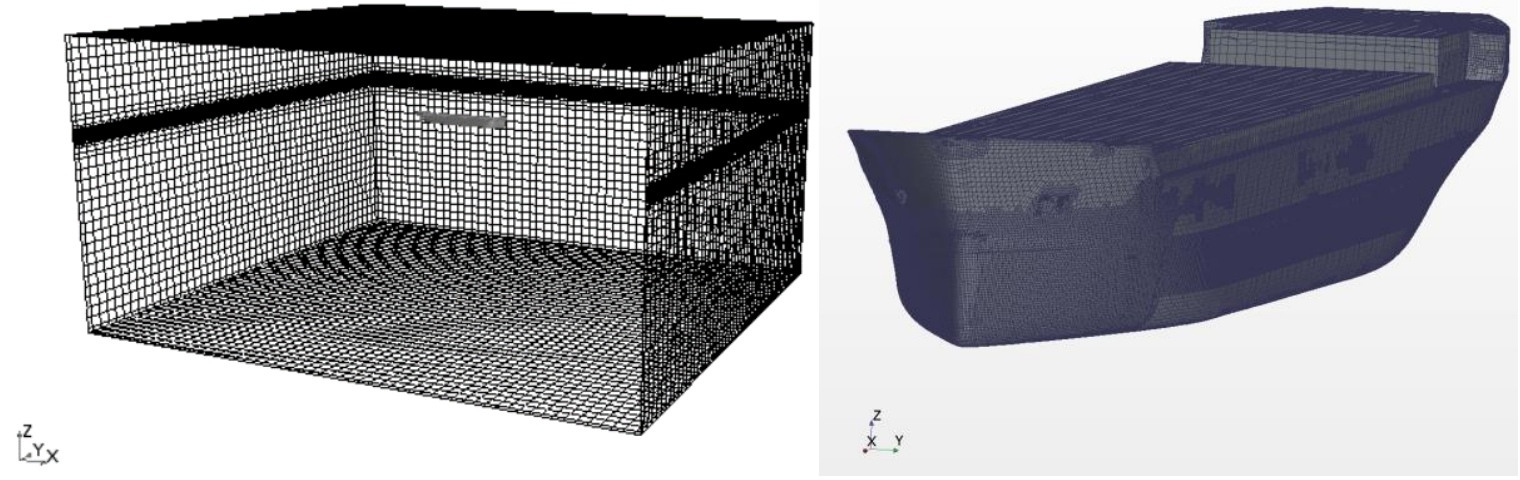

Figure 6 Fluid domain (left) and bow refinements (right)

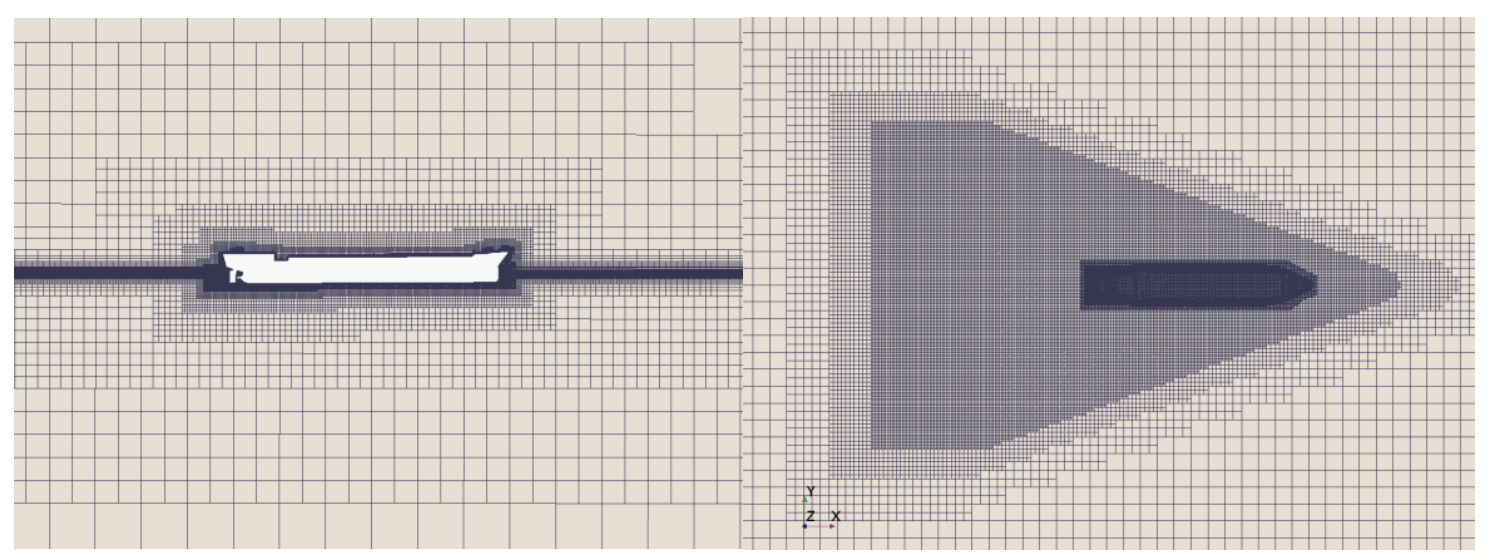

Figure 7 Mesh in the region of free-surface and wake (right plan view, left represents a side view of the mesh)

\section{Mesh Performance Analysis}

A mesh independence study was conducted for the simulation set-up on four different grid resolutions by varying the mesh size input parameter while holding all other parameters constant and following recommended practices (ITTC, 2017). The uniform parameter refinement ratio was established as $\sqrt{2}$. As a result, four grids are generated as follows, the coarse grid (G.1) with 12.3 million cells, the medium grid (G.2) with 25.4 million cells, the fine grid (G.3) with 41.1 million cells and the finest grid (G.4) with 62.5 million cells. Table 5 reveals the results for the total resistance and viscous resistance coefficients $\left(\mathrm{C}_{\mathrm{T}}\right.$ and $\mathrm{C}_{\mathrm{v}}$, respectively) for grids $\mathrm{G} 1, \mathrm{G} 2, \mathrm{G} 3$ and $\mathrm{G} 4$ together with the convergence ratio $\varepsilon_{\mathrm{ij}}$ between grids. Overall, it can be seen that monotonic convergence is achieved as the mesh resolution grows. $\varepsilon$ reveals a ratio of $0.44 \%$ for $\mathrm{C}_{\mathrm{T}}$ and $0.6 \%$ for $\mathrm{C}_{\mathrm{v}}$ between meshes 3 and 4 . 
Also, mesh convergence is monitored by plotting the resolved Turbulent Kinetic Energy (TKE) convergence for the four grids at FR1 as shown in Figure 8. In this case, resolved TKE has been chosen as this quantity is very relevant to predict the bilge vortex physics fully. It can be seen that G3 and G4 are able to calculate a similar amount of TKE contained in the flow whereas G1 and G2 significantly diverge from the results; thus, suggesting that in G1 and G2, the bilge vortex falls in the RANS or shielding region. Nevertheless, this assumption will be discussed in more depth through the present section. Overall, the convergence of the resistance coefficients together with the resolved turbulent kinetic energy suggests that the current set-up has reached a convergence.

Table 5 Mesh independence study for simulation at 8 knots

\begin{tabular}{lllllllll}
\hline \multicolumn{2}{c}{ Grid } & G1 & G2 & G3 & G4 & & & \\
& $\mathrm{y}+$ & 1.4 & 1.1 & 0.9 & 0.7 & & & \\
\hline Parameter & Benchmark data & & & & & $\varepsilon_{12} \%$ & $\varepsilon_{23} \%$ & $\varepsilon_{34} \%$ \\
\hline $\mathrm{C}_{\mathrm{T}} \times 10^{-3}$ & - & 2.09 & 2.16 & 2.25 & 2.24 & 3.35 & 4.17 & -0.44 \\
& $\mathbf{2 . 2 3}$ (Workshop) & - & - & - & - & - & - & - \\
& CFD\%Workshop & -6.27 & -3.24 & 0.88 & 0.44 & - & - & - \\
$\mathrm{C}_{\mathrm{v}} \times 10^{-3}$ & - & 1.79 & 1.72 & 1.67 & 1.66 & 3.91 & 2.89 & 0.60 \\
& $\mathbf{1 . 6 2}$ (ITTC57) & - & - & - & - & - & - & - \\
& CFD\%ITTC & 10.49 & 5.81 & 3.01 & 2.41 & - & - & - \\
\hline
\end{tabular}

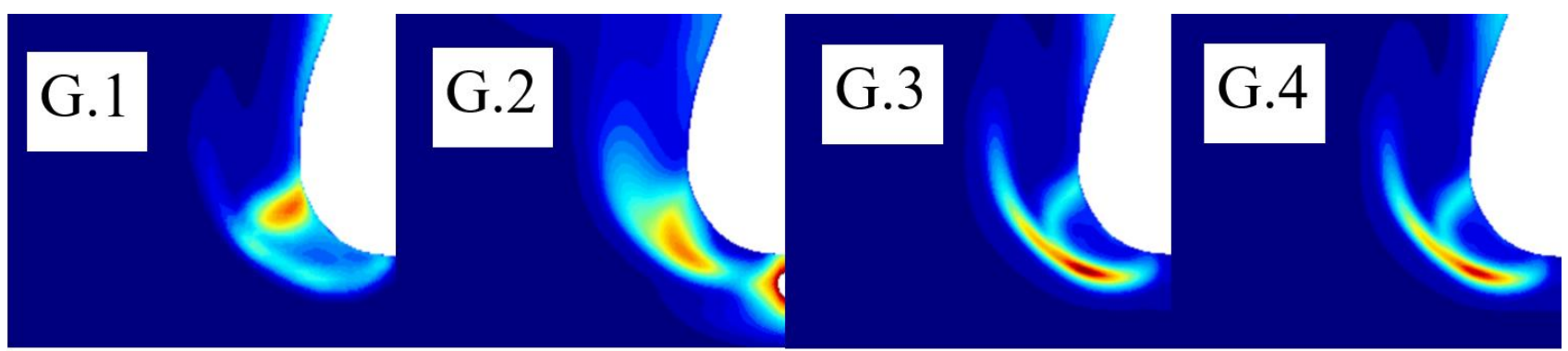

Mean of Resolved TKE

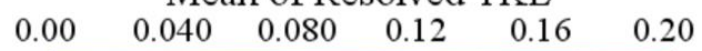

Figure 8 Mean resolved TKE for G1, G2, G3 and G4

Table 5 also compares the $\mathrm{C}_{\mathrm{T}}$ simulation results for each grid versus the mean $\mathrm{C}_{\mathrm{T}}$ value obtained during the 2016 Full-Scale Ship Hydrodynamics Workshop (Lloyds Register, 2016). Note that the comparison error $\left(C F D \%\right.$ Workshop $\left.=100\left(C_{T_{C F D}}-C_{T_{\text {Workshop }}}\right) / C_{T_{\text {Workshop }}}\right)$ takes values of 
around $1 \%$ for $\mathrm{G} 3$ and $\mathrm{G} 4$ respectively suggesting that the simulation results are in line with previous investigations.

Besides, the calculated $\mathrm{C}_{\mathrm{v}}$ is compared to the ITTC57 (ITTC, 1957) established friction lines, as shown in Table 5. First, it can be seen that $\mathrm{C}_{\mathrm{v}}$ CFD results are above the ITTC57 friction lines for all the grids and with a deviance of approximately $+3 \%$ for G3 and G4. This indicates that the near-wall turbulence and calculated shear stress are in the acceptable range. On the other hand, the fact that the $\mathrm{C}_{\mathrm{v}}$ decreases as the mesh resolution increases could have to see with the fact that the LES region is approaching the wall. In particular, some studies have reported Modelled Stress Depletion or MSD issues when implementing an IDDES approach (Ashton, 2016) which manifest as a difficulty to reproduce the turbulence through the interface between RANS and LES zones in the attached regions (Spalart et al., 1997). MSD is seen in cases when the LES length scale drops below the RANS length scale close to the wall region, triggering the numerical model into LES mode close to the wall. In such a scenario it is possible that a close to the wall LES region is not refined enough for a wall-resolved LES, triggering a turbulence depletion in the near-wall region. Some studies have reported that the MSD issue might be particularly strong for overly refined grids (Ashton, 2016). However, as the IDDES calculated $\mathrm{C}_{\mathrm{v}}$ is well above the ITTC57 friction line, this might not be an issue for the present work.

In order to further confirm that the simulations do not present significant stress depletion, modelled and resolved turbulence components are separated and analysed. Figure 9 shows the mean modelled turbulent kinetic energy (TKE) at a longitudinal plane LG1 1/2. Overall, it is possible to differentiate a detached region (in dark blue) where no turbulence has been modelled. Additionally, the figure displays a near-wall region (zoomed) which indicates that turbulent kinetic energy is present in the near-wall region and suggesting that RANS mode is well acting in the near-wall region. On the other hand, Figure 10 indicates that the turbulent kinetic energy contained in the bilge vortex has been resolved as it was desired. The zoomed region represents an isometric view of the resolved TKE at FR1, where it is possible to corroborate that most of the turbulence contained in the bilge vortex has been resolved. Note that the scale of TKE is different for the modelled and resolved turbulence plots. 


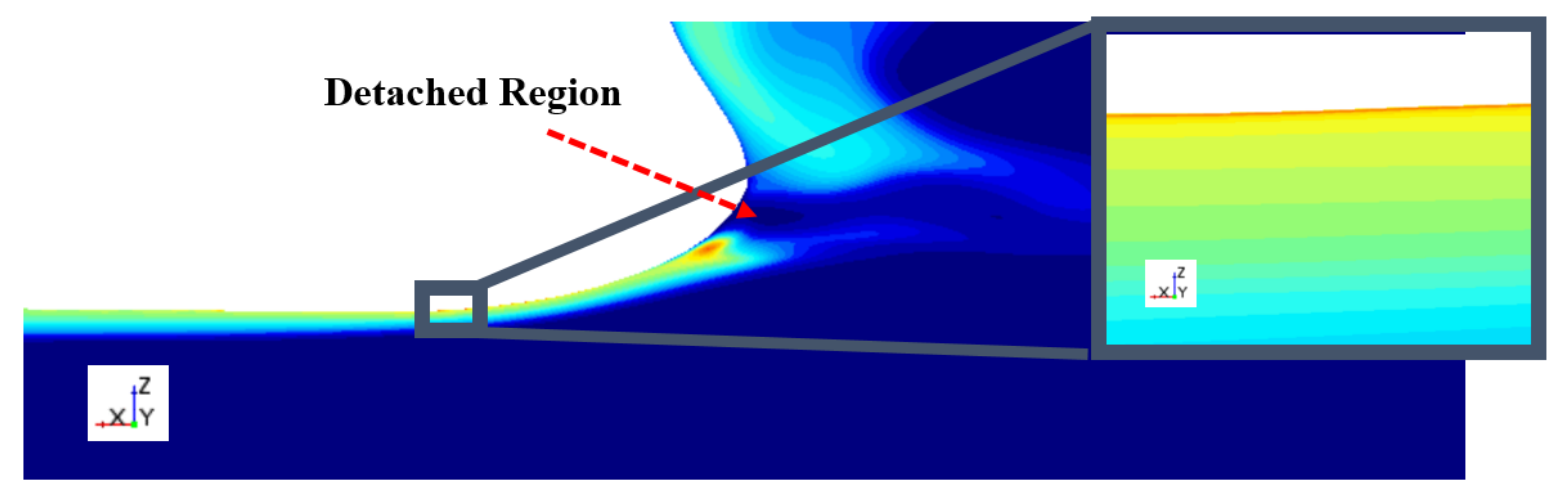

Mean of Modelled TKE (J/Kg)

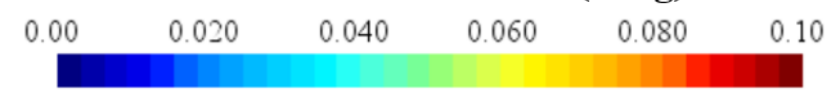

Figure 9 Modelled TKE at LG1 1/2

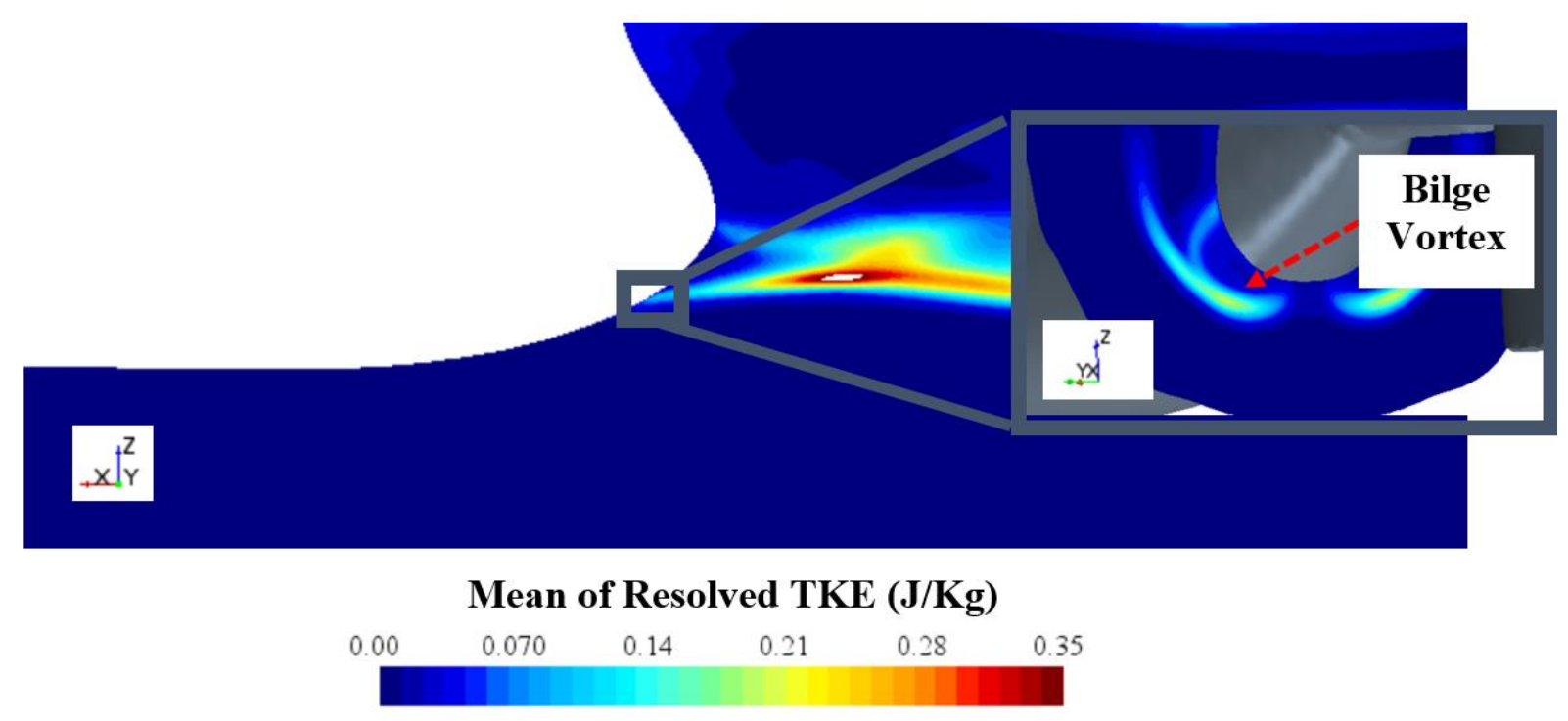

Figure 10 Resolved TKE at LG1 1/2 (Zoomed at FR1)

The next check before accepting the mesh configuration is to confirm that the bilge vortex is resolved and that as desired it falls in the LES region. Figure 11 (left) represents the IDDES blending factor, which shows the limits of the RANS and LES zones, whereas Figure 11 (right) displays the mean resolved TKE at FR1. The comparison demonstrates that as desired, the core of the bilge vortex falls in the LES region and confirms that the present grid will resolve most of the vortex turbulence. This check was also conducted at several planes downstream towards the propeller region to ensure a proper resolution of the large vortex structures. At this stage, it could still be argued that further refinements could be beneficial to ensure that a greater extent of the bilge vortex falls in the LES region. However, this is questionable because refining the mesh could trigger the LES region to approach the hull surface at the aft of the ship. Looking back at Figure 11, it could be seen that the LES region is already close to the near-wall region, so it could be risky to significantly reduce the 
mesh size, since there could be an incursion of the LES region into the RANS region and thus trigger the undesired MSD phenomenon.

From this analysis, it is clear that a thorough mesh performance analysis like the one conducted as part of this work is required and recommended to verify the shielding section location. In this case, the mesh is proved to be adequate for further analysis.

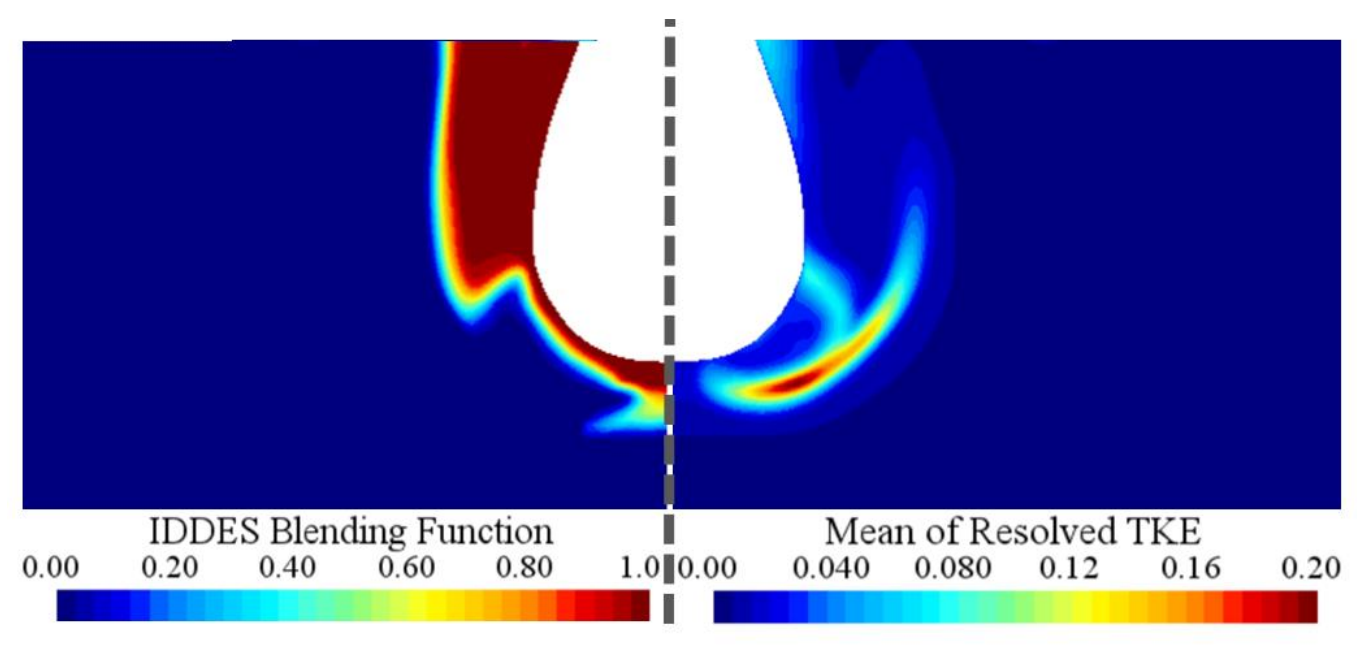

Figure 11 IDDES blending function vs resolved TKE at FR1

\section{Results Analysis}

The numerical results of the flow around the hull are shown and discussed in terms of ship resistance coefficients, limiting streamlines, nominal wake and the boundary layer at the stern of the ship. The results of this assessment are referred to the following parameters:

- Distances (x, y and z) are dimensionalised as a function of the ship length (L), beam (B) and draught $(\mathrm{T})$ as follows: $\mathrm{x} / \mathrm{L}, \mathrm{y} / \mathrm{B}$ and $\mathrm{z} / \mathrm{T}$.

- The incoming flow, $\overrightarrow{U_{\infty}}$, is in the negative x-direction.

- The components of mean velocity in the (x, y and $\mathrm{z}$ ) directions are denoted by $\overline{\vec{u}}=$ $\left(\bar{u}_{x}, \bar{u}_{y}, \bar{u}_{z}\right)$,

- Velocity is dimensionalised as a ratio of the freestream velocity $\overrightarrow{\vec{u}}=\left(\frac{\bar{u}_{x}}{U_{\infty}}, \frac{\bar{u}_{y}}{U_{\infty}}, \frac{\bar{u}_{z}}{U_{\infty}}\right)$.

\subsection{Ship Resistance, Pressure and Shear Viscous Coefficients}

Figure 12 shows the bare hull pressure and shear resistance coefficients per unit length (distribution along the hull). Each unit length is represented by a strip, where the $1^{\text {st }}$ and $14^{\text {th }}$ strips correspond to the aft and bow respectively. 
Looking at the plot shown in Figure 12 (left), it is possible to see that as the flow approaches the bow $\left(14^{\text {th }}\right.$ strip), the pressure coefficient sharply decreases, establishing a favourable pressure gradient. From the $10^{\text {th }}$ to the $6^{\text {th }}$ strip, the pressure remains almost constant along the length of the ship. In the central part of the ship, the pressure coefficient is rather small. By contrast, from the $6^{\text {th }}$ strip towards the aft end of the ship, the pressure quickly builds-up establishing an adverse pressure gradient. This pressure coefficient trend was expected, and it is in line with previous investigations (Patel et al., 1990, Larsson et al., 2010). The total pressure, viscous and resistance coefficients integrated over the whole hull are given in Table 6.

Table 6 Integral Hydrodynamic Resistance Coefficients

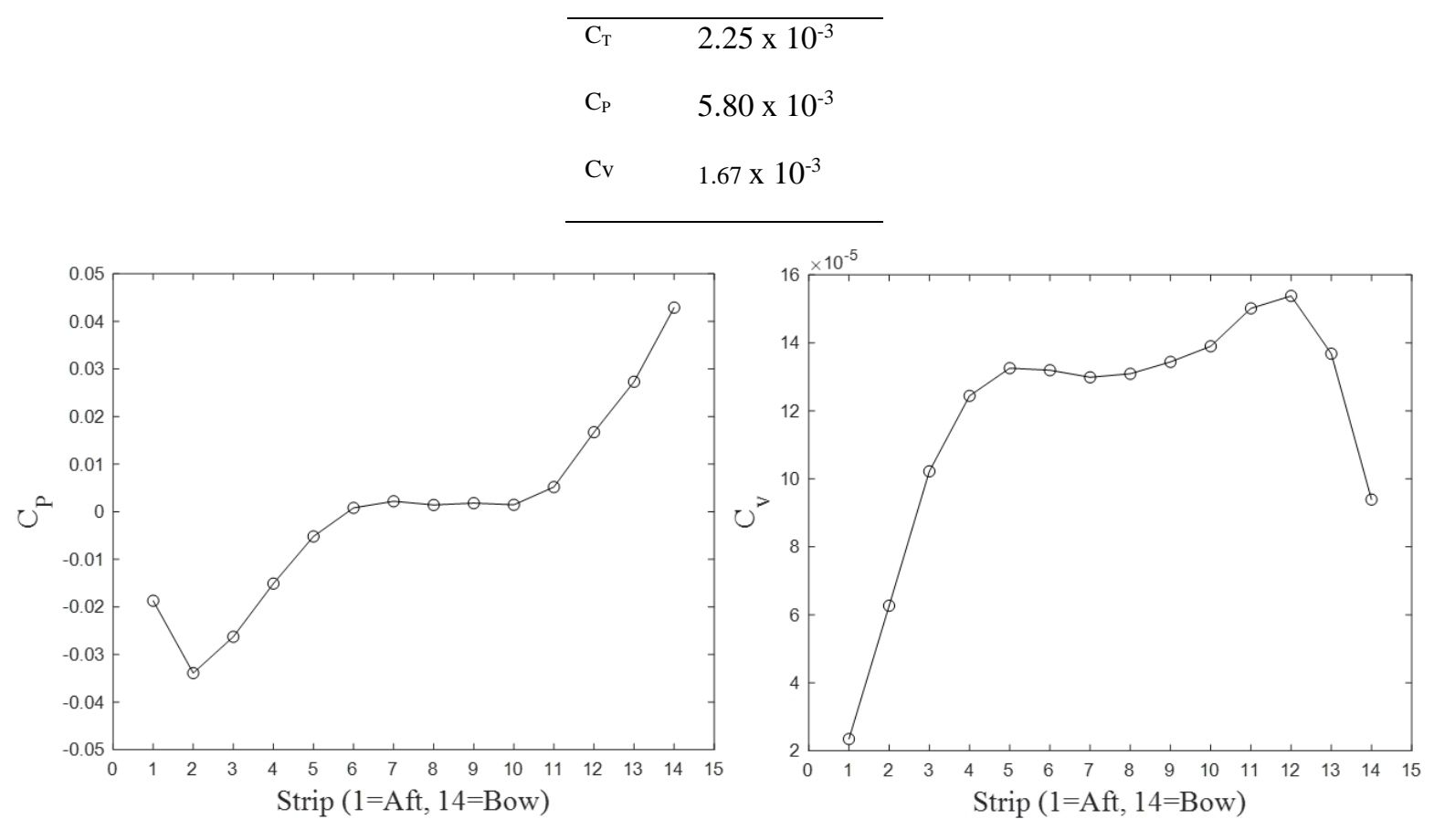

Figure 12 Pressure and viscous resistance coefficient comparison per unit length

Overall, the pressure resistance displays a significant pressure imbalance between the aft and bow ends of the ship. At the bow, the pressure resistance coefficient reaches values of approximately 0.045 , whereas at the stern, the pressure resistance coefficient decreases reaching a value of just 0.018. A small pressure resistance imbalance is expected, and generally, it implies an extra drag component that negatively impacts on the hydrodynamic performance of the ship. However, a strong pressure imbalance between the fore and aft ends of the ship could also indicate a loss of hydrodynamic efficiency due to the existence of hull flow separation.

A closer explanation of the hull pressure variations can be seen in the dynamic pressure coefficient $C_{P d}$ distribution along the ship hull (Figure 13). Each series represents a ship waterline $\left(\mathrm{WL}_{\mathrm{i}}\right)$ ranging from WL1 (near the water surface) to WL4 (near the keel). $C_{P d}$ is defined as $C_{P d}=\frac{2\left(P_{T}-P_{h}\right)}{\rho{\overrightarrow{U_{\infty}}}^{2}}$, where 
$P_{T}$ is the total pressure and $P_{h}$ is the hydrostatic pressure. It is essential to notice that a $\frac{d C_{P d}}{d x}>0$ indicates the existence of an adverse pressure gradient that tends to slow down the shear layer flow. This is particularly problematic for the near-wall region flow which contain the least of momentum (low velocity of the fluid particles) due to the proximity to the wall.

Figure 13 confirms that the streamwise pressure gradient is initially favourable at the bow $(\mathrm{x} / \mathrm{L}=1)$, meaning that the fluid particles are accelerated. Further downstream, the pressure coefficient becomes slightly adverse towards the parallel body ( $\mathrm{x} / \mathrm{L}$ between 0.67 to 0.29 ) and significantly adverse towards the stern of the $\operatorname{ship}(\mathrm{x} / \mathrm{L}=0)$. The region towards the end of the ship is therefore affected by a negative pressure gradient meaning that the fluid particles are decelerated. As a primary consequence of the aft pressure gradient, it is expected to see a sudden longitudinal direction deceleration on the near-wall fluid particles within the aft end boundary layer.

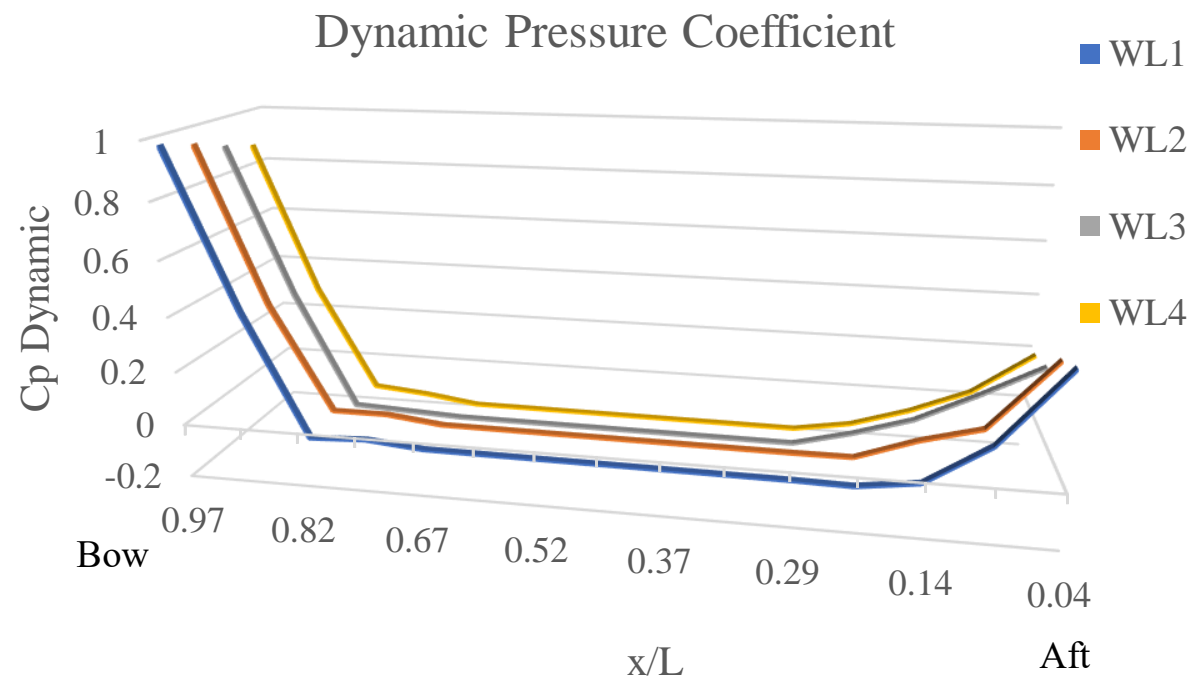

Figure 13 3D Plot showing the dynamic pressure gradient along the ship length at different waterlines

Flow separation induced by a strong aft and bow end pressure imbalance was confirmed after plotting the hull limiting streamlines along the stern of the ship (Figure 12). A streamline has been defined as a line which is parallel to the local velocity vector $\overline{\vec{u}}=\left(\bar{u}_{x}, \bar{u}_{y}, \bar{u}_{z}, t\right)$ over the hull surface (White, 2011). Overall, the streamlines travel over the parallel body displaying little lateral curvature and bending upwards (1) as they pass over the aft end shoulder. This behaviour is consistent with the dynamic pressure distribution over the hull. It can be seen in Figure 13 that there are no pressure differences on the parallel body, driving the flow to travel straight. By contrast, the pressure is higher near the keel and lower closer to the free-surface, pushing the flow to travel upwards as seen in Figure 14. Further downstream, the streamlines curve sharply downwards (3), abruptly converging closer to the aft stagnation point (4). Thus, a vortex sheet (5) detaching from the hull surface is created because 
of the strong near-wall streamline convergence, as seen in Figure 14. Further analysis of threedimensional streamlines along the hull could be alternatively used to confirm the findings explained above.

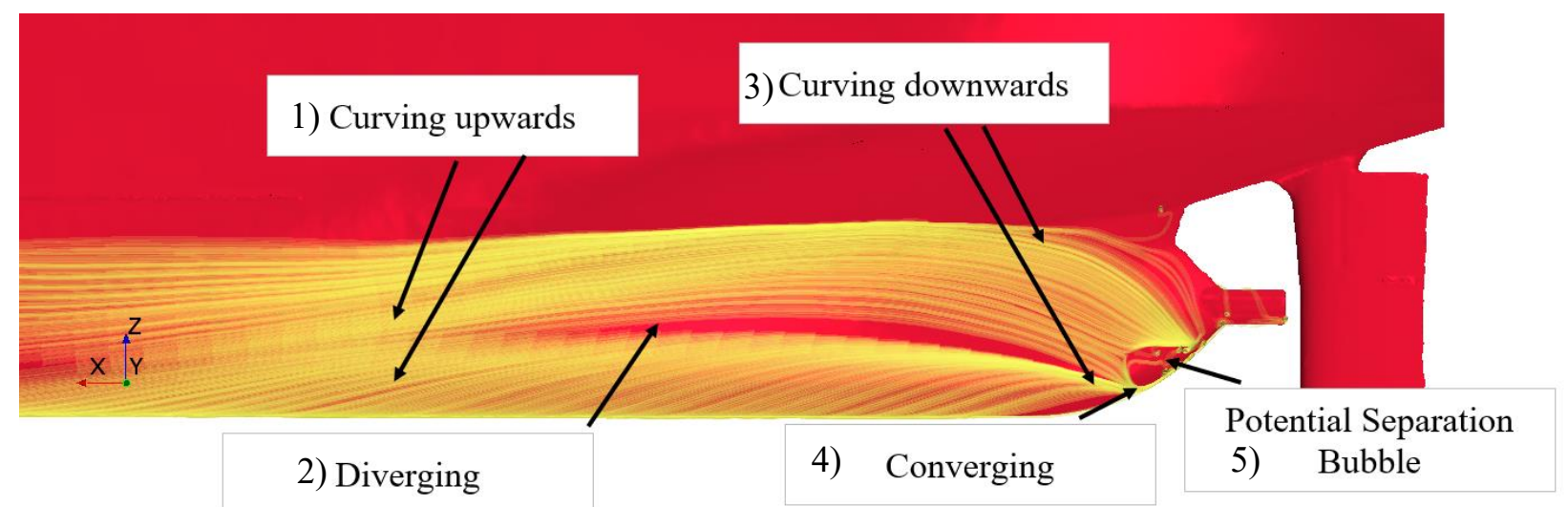

Figure 14 Limiting streamlines over the aft end of the hull

The detached shear layer is therefore expected to be seen at the propeller nominal wake region that is displayed in Figure 15. The nominal wake measurements were taken at the propeller plane (FR1/2) which are represented by mean normalised axial speed over the freestream velocity $\left(\frac{\bar{u}_{x}}{\bar{U}_{\infty}}\right)$. Red/orange regions indicate the existence of slow fluid, whereas blue areas suggest a flow velocity close to the main freestream. An intense hook-shape in the wake field is identified and located at approximately 5 o-clock and the 7 o-clock positions. This hook-shape is caused by a strong bilge vortex that typically forms behind the stern of ships with small bilge radius. The image confirms the existence of a reversed flow upstream of the propeller (red areas). The high variation in the non-dimensional axial velocity measurements suggests that the vessel may be suffering from blade load fluctuations and possibly propeller vibrations. Additionally, the nominal wake velocity field shows that the flow separation on the propeller plane occupies a great extent of the total nominal wake disk, causing a notable detriment on the propeller's hydrodynamic performance. 


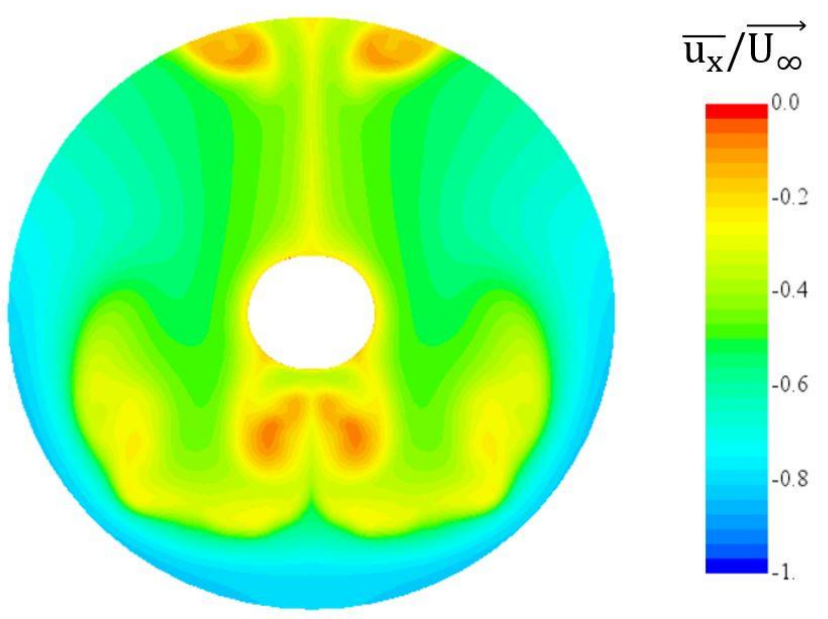

Figure 15 Mean Nominal Wake Field at FR1/2 (x/L=0.02)

The presence of the strong bilge vortex and the detached shear layer is expected to be reflected in the viscous resistance distribution shown in Figure 12 (right). As the flow approaches the fore end of the ship, the viscous drag grows rapidly until the $12^{\text {th }}$ strip. From the $10^{\text {th }}$ to the $4^{\text {th }}$ strip $\mathrm{C}_{\mathrm{V}}$ remains almost steady whereas, at the aft end, $\mathrm{C}_{\mathrm{V}}$ rapidly decreases. The rapid decrease on $\mathrm{C}_{\mathrm{V}}$ was expected, and it is mainly due to the presence of a thick boundary layer together with the reversed flow shown in Figure 15.

A more detailed investigation of the shear stresses can give a better insight into the flow behaviour, as shown in Figure 16. It is important to notice that $C_{V}$ is calculated based on the shear stress tensor. The figure displays that the viscous coefficient is at its lowest value over the upper part of the bow. The lower coefficient is the result of flow stagnation. By contrast, the viscous coefficient is at its highest value over the foremost part of the keel. Further downstream over the parallel body, the viscous coefficient remains practically unchanged. It is important to note that the parallel body region has a shallow curvature, and the boundary layer would be expected to behave similarly to a flat plate. On the other hand, the aft end hull surface presents a large curvature, and this is reflected on $C_{V}$. At the aft end bilges $C_{V}$ increases due to the action of the bilge vortex. Contrariwise, the near-wall flow rapidly loses momentum and $C_{V}$ sharply decreases along the aft end shoulder. Consequently, the shear velocity is much smaller over the aft end than over the bow. Also, near the propeller hub, the viscous coefficient approaches zero; thus, reversed flow is again confirmed. 

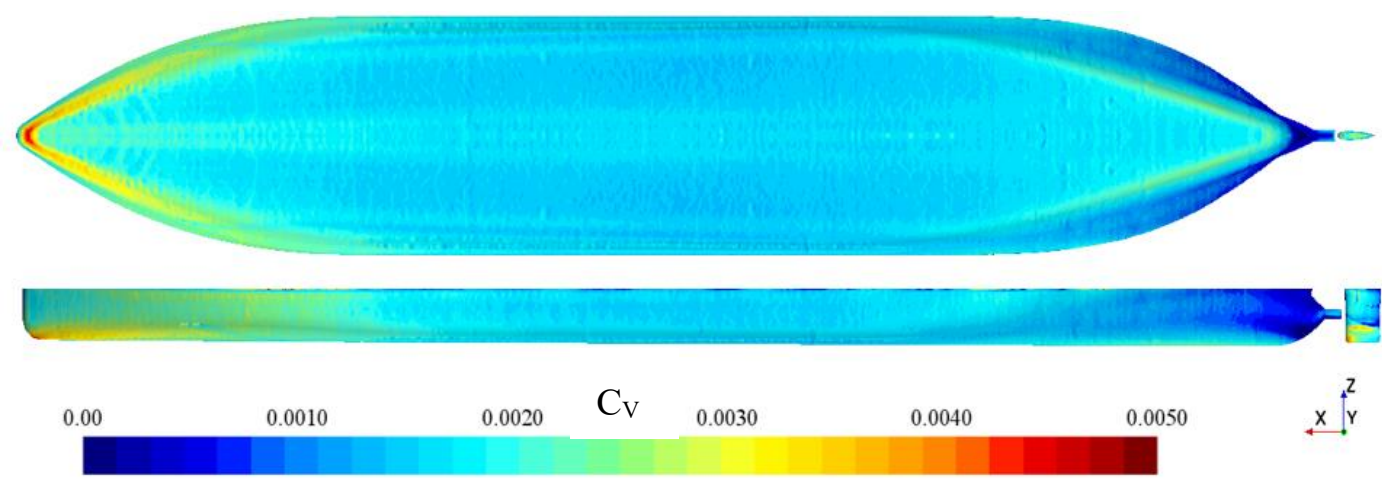

Figure 16 Time-averaged viscous coefficient along the hull

\subsection{Boundary Layer Velocity Profiles}

The evolution of the boundary layer velocity fields at different cross-sections or frames (FRi) is displayed in Figure 17. As expected, the diminishing hull cross-section caused a thickening of the boundary layer in the stern region and the convergence of the streamlines shown in Figure 12. Additionally, a 'bulge' is formed, which grows further downstream. This bulge is shown in Figure 17, and its origin and trajectory are tracked in Figure 18. Its origin is thought to be formed by the 'squeeze' effect on the flow from the bilge vortex near the keel and moving downstream towards the propeller Figure 18. The bulge shape has been previously shown in Larsson and Raven studies (2010).

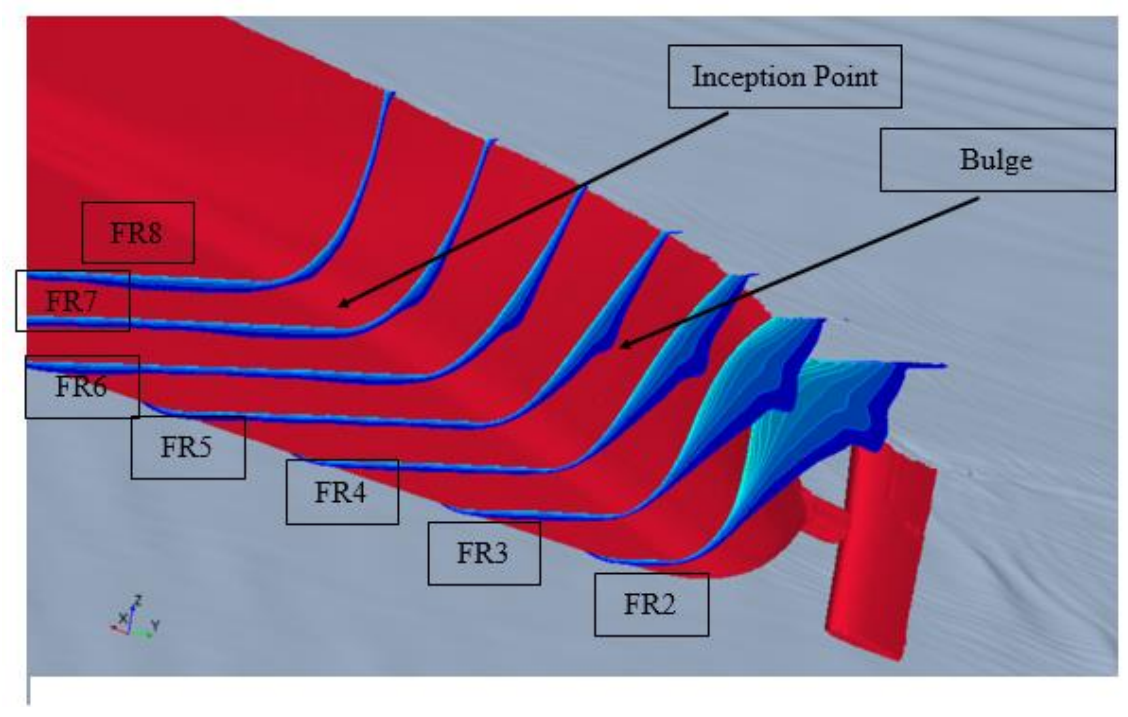

Figure 17 Boundary layer thickening phenomenon given by plotting $0.99 \mathrm{U}$ 


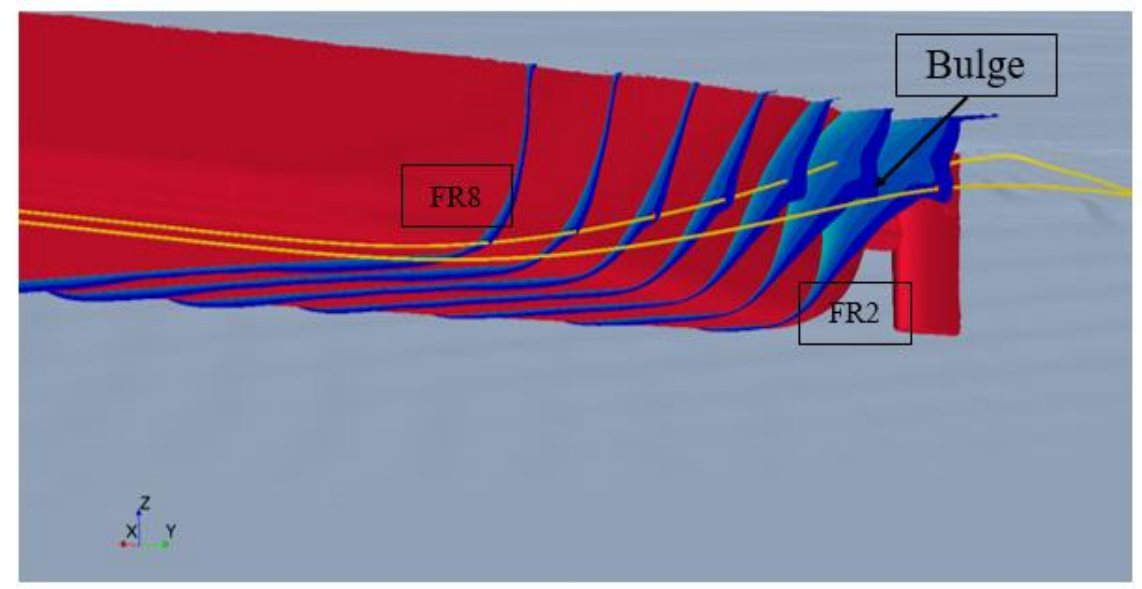

Figure 18 Streamlines passing by the bulge

With a better understanding of the overall hydrodynamics of the ship, it is appropriate to study the velocity distribution in several cross-planes on the aft end body of the ship. Figure 19 to Figure 22 show the iso-velocity contours at 4 different frames along the ship hull length. Velocity contours are given for the dimensionless axial velocity component. Also, tangential velocity components vectors are displayed as well. At the foremost station (FR 10) shown in Figure 19, it is possible to see the existence of a relatively thin boundary layer with no significant crossflows. This station was positioned at the end of the parallel body, and as expected, it displays a boundary layer that is evenly distributed around the girth. Downstream at FR7 (Figure 20), the boundary layer considerably thickens, and stronger crossflows appear. These crossflows were again anticipated. They appear due to the bilge vortex action together with the intense pressure field over the hull surface. The flow turns downwards near the free-surface, whereas at the bottom of the ship, the flow goes upwards. This phenomenon is consistent with the near-wall streamlines path shown in Figure 12.

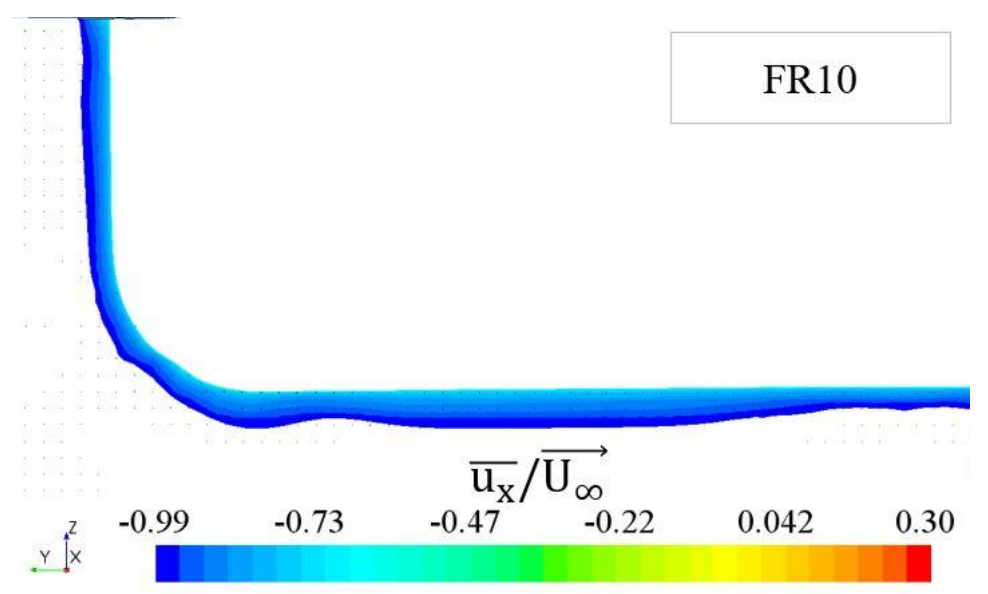

Figure 19 Mean velocity field at the end of the parallel body at FR10 


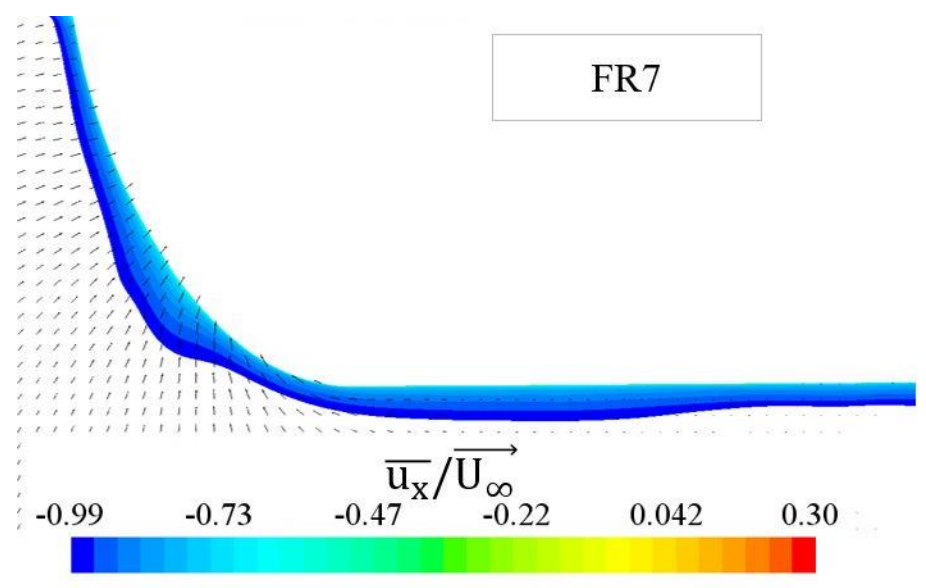

Figure 20 Mean velocity field at FR7

Further downstream, at FR3 (Figure 21), a considerable redistribution of the boundary layer flow has occurred. At first, it is possible to observe a rapid thickening of the outer boundary layer region, which in turns indicates strong streamlines converge. By contrast, the near-wall flow boundary layer region becomes thinner. This phenomenon is in line with the streamlines divergence shown at middraft of Figure 14. Also, at mid-draft, the crossflow vectors show a new co-rotating vortex that suddenly appears on the scene due to curvature change (from convex to concave) of the hull surface. This vortex, typically called the side vortex, is weak and is merging with the bilge vortex further downstream (FR1, Figure 22).

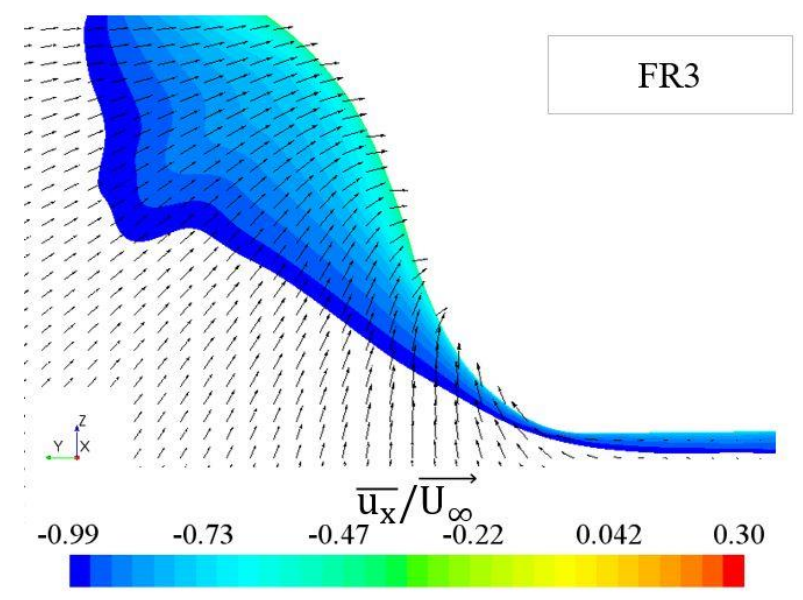

Figure 21 Mean velocity field at FR3

At the last station, FR1 (Figure 22 left), the same boundary layer features are seen. There is a clear thinning and thickening of the inner and outer regions of the boundary layer, respectively. There is an 'island' of reversed flow near the hub. This region is represented in orange, and it represents a detached shear layer where $\bar{u}_{x} / \overrightarrow{U_{\infty}}>0$. The reversed flow is enhanced by the bilge vortex, and it is the 
main contribution of the strong pressure resistance imbalance shown in the resistance coefficient distribution (Figure 22 right).
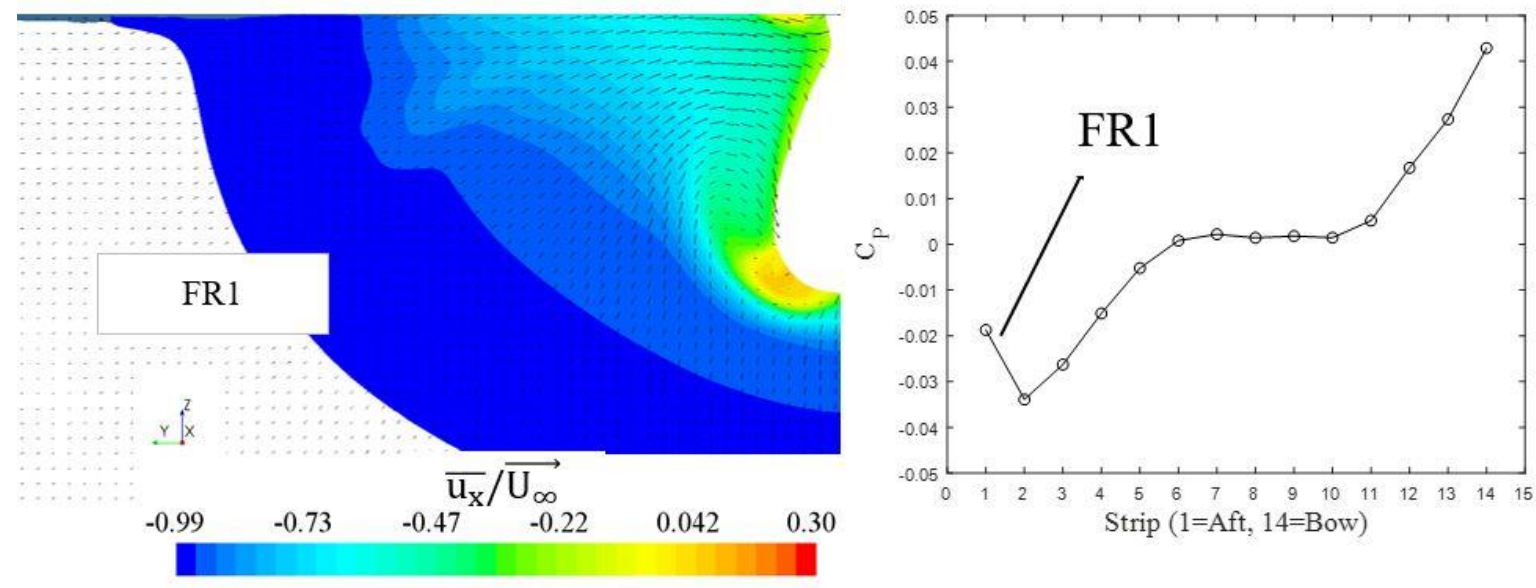

Figure 22 Mean velocity field at FR1

It was found insightful to further explore the underlying physics behind the thinning of the boundary layer around the keel and the boundary layer thickening around the hollow at mid-girth that was shown at FR3 (Figure 21). An explanation of this phenomenon can be found on the boundary layer turbulent kinetic energy distribution Figure 23. At FR10 (Figure 23 left), the turbulent kinetic energy is evenly spread across the cross-section. Downstream, as the boundary layer thickens, the turbulent kinetic energy distributes across the new thicker shear layer. Close to the keel, the turbulent kinetic energy remains in the near-wall region and additional turbulent energy is produced due to the action of the bilge vortex. Therefore, the bilge vortex effect contributes to withstand the aft end negative pressure gradient.

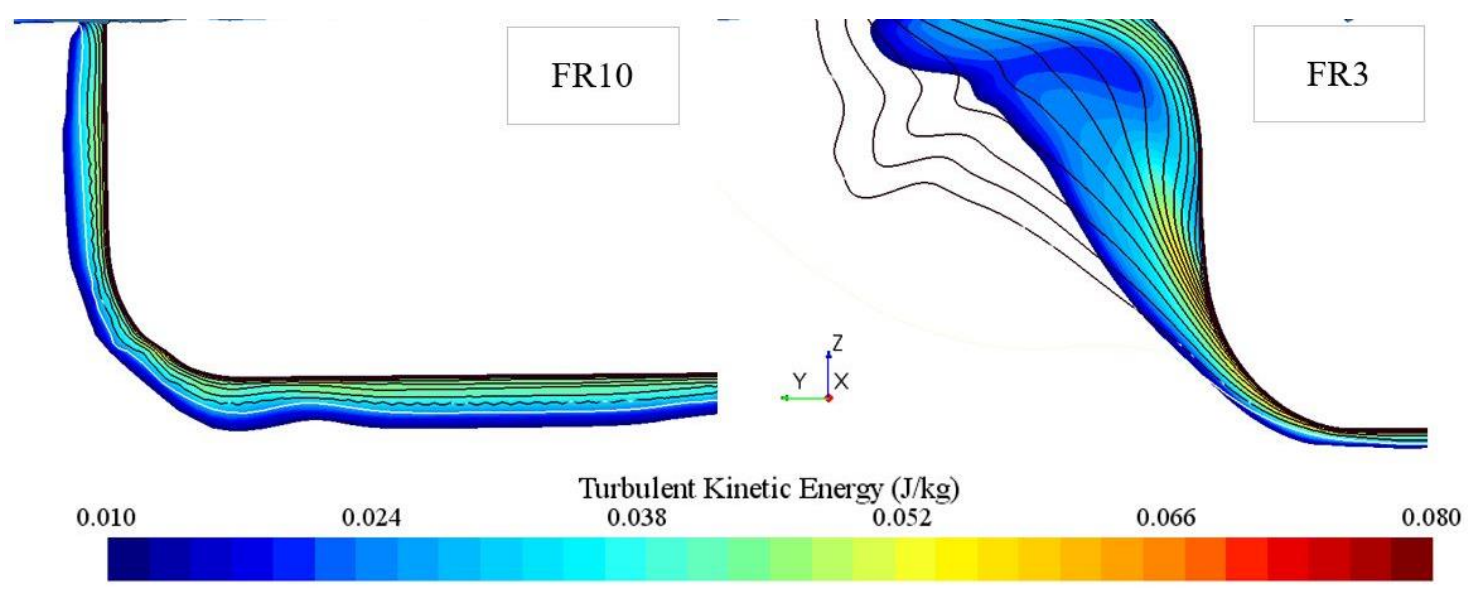

Figure 23 Turbulent kinetic energy distribution. Dark isolines show the boundary layer extension at that crosssection. 
The previous in-depth analysis has served to describe the boundary layer behaviour. A more detailed evaluation requires a more meticulous analysis of the velocity profiles at each probe point.

Velocity profiles at the probe points are extracted and shown in Figure 24 as a function of the mean velocity magnitude and the wall distance: $\mathrm{y}_{+} \mathrm{vs} u_{+}=f\left(y_{+}\right)$. Each plot represents a frame (FRi) intersection with waterlines (WLi). For instance, the FR10 plot and WL1 series represent the velocity profiles measured on the probe point located at the intersection of the frame 'FR10' and the waterline 'WL1' (or the point PFR10WL1). For presentation purposes, the flat bottom of the ship is not shown since the boundary layer behaviour resembles that of a flat plate with no flow detachment or thickening phenomena.

Figure 24 also includes the theoretical $u_{+}=y_{+}$and the Von-Karman universal log-law expressions for a smooth surface, $u_{+}=2.5 \ln \left(y_{+}\right)+5$ (Schlichting et al., 2017). The flow measurements are referred to a local coordinate system where the origin was located at the hull surface at the desired probe point. The local y-axis coordinate system was defined normal to the hull surface whereas the local $\mathrm{x}$-axis was parallel to the undisturbed freestream flow.

The first plot, FR10, shows that the velocity profiles in the viscous sub-layer and log-law layer agree well with the theoretical lines at the four WLi's. The velocity profiles distribution does not vary across the hull height. This trend was expected, and it is a consequence of the small curvature change that the hull plate presents towards the parallel body.

Further downstream (from FR7) the effects of the adverse strong pressure gradient become clearer. At greater distances from the hull surface, the mean $u_{+}$profile velocity exceeds the classical logarithmic law-of-the-wall line in a wake-like shape, known as the outer boundary layer region or wake (as seen on the FR3 plot). Further downstream (at FR3 and FR2) the boundary layer rapidly grows in thickness as a reaction to the adverse pressure gradient which in turn further retracts the extent of the log-law region and increases the size of the wake region. It was noticeable that moving downstream; the velocity profiles deviate significantly from the standard log-law. Similar behaviour has been seen by $\mathrm{Na}$ et al. (1998), who conducted DNS simulations and experiments over plates subject to flow separation. 
FR10

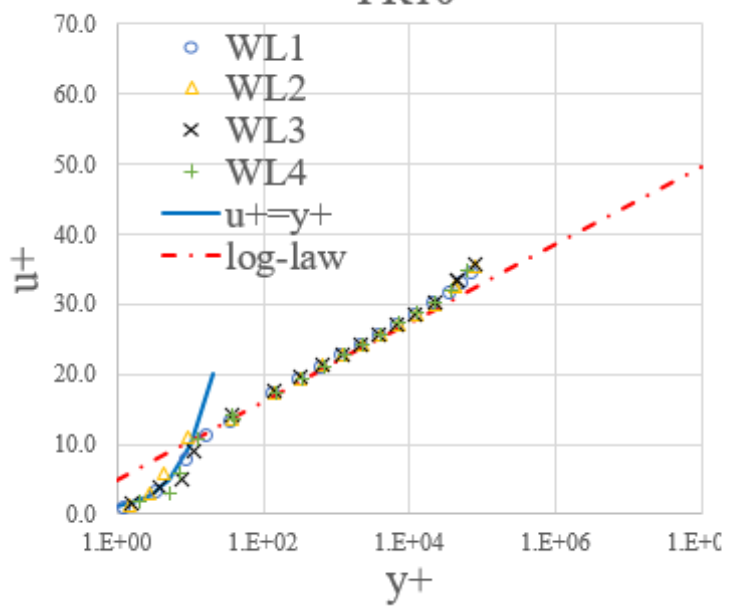

FR3

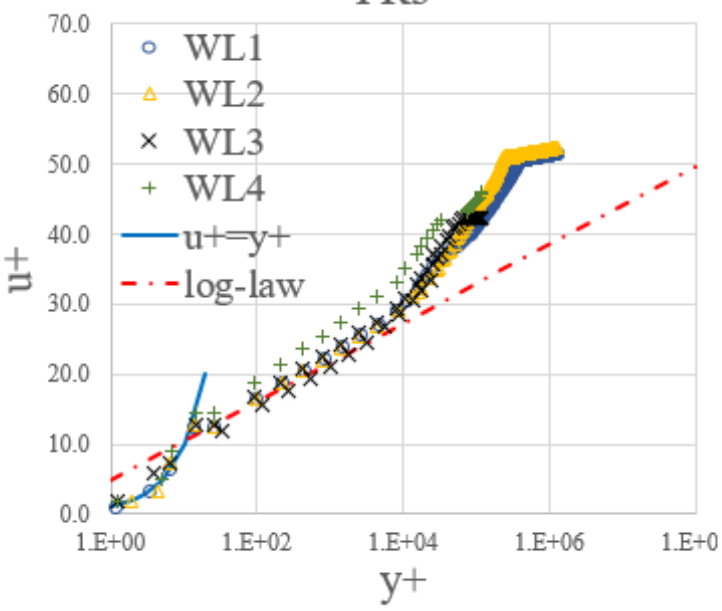

FR7

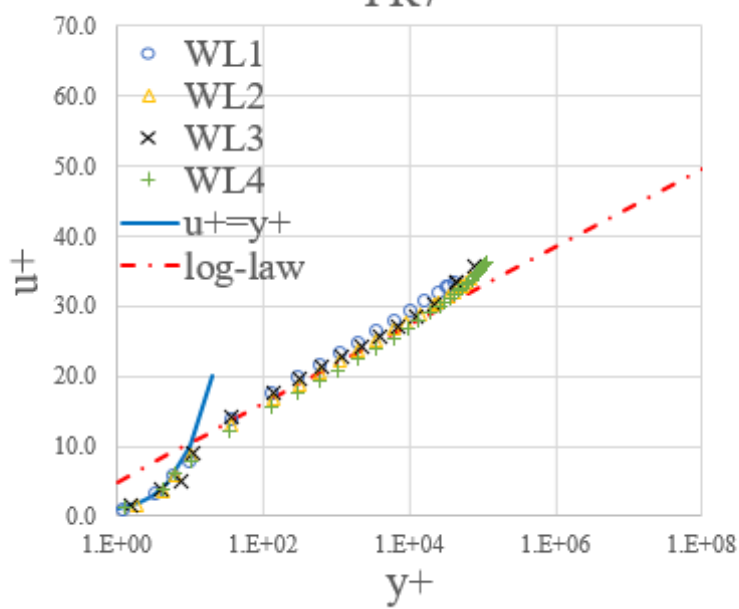

FR2

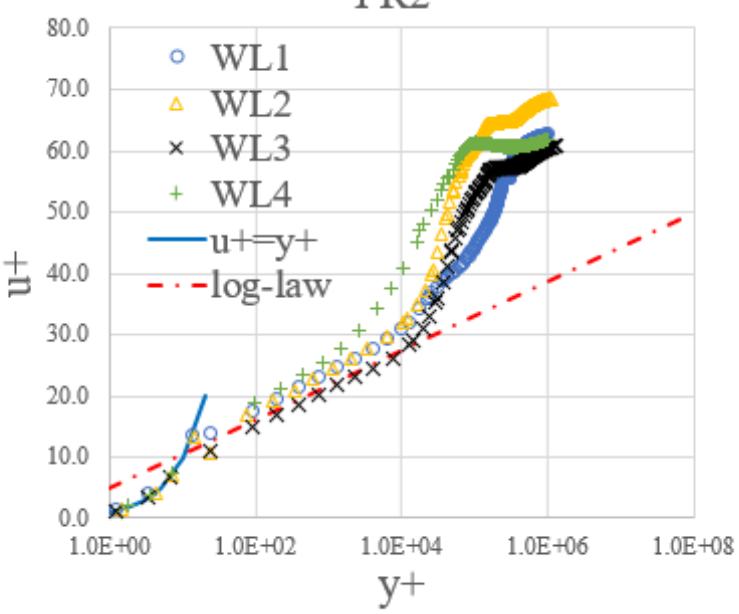

Figure $24 \mathrm{u}_{+} / \mathrm{y}_{+}$velocity profiles across the hull length

Closer to the propeller, at FR1 (Figure 25), the boundary layer structure dramatically varies, and flow separation is displayed. It can be seen that the green waterline WL4 (closer to the keel) $u_{+}$values spike. This phenomenon is expected since the shear velocity $u_{\tau}$ is approaching a value of 0 at the detachment point. The flow separated region is further confirmed by plotting isosurfaces of negative shear stress as displayed in Figure 26 (in blue) which corresponds to the probe point PFR1WL4.

The boundary layer velocity profiles shown within this work can be further used to obtain the boundary layer displacement thickness, momentum thickness or shape factor that could be useful to determine the exact location where the boundary layer loses the capability to withstand the adverse pressure gradient effects. 


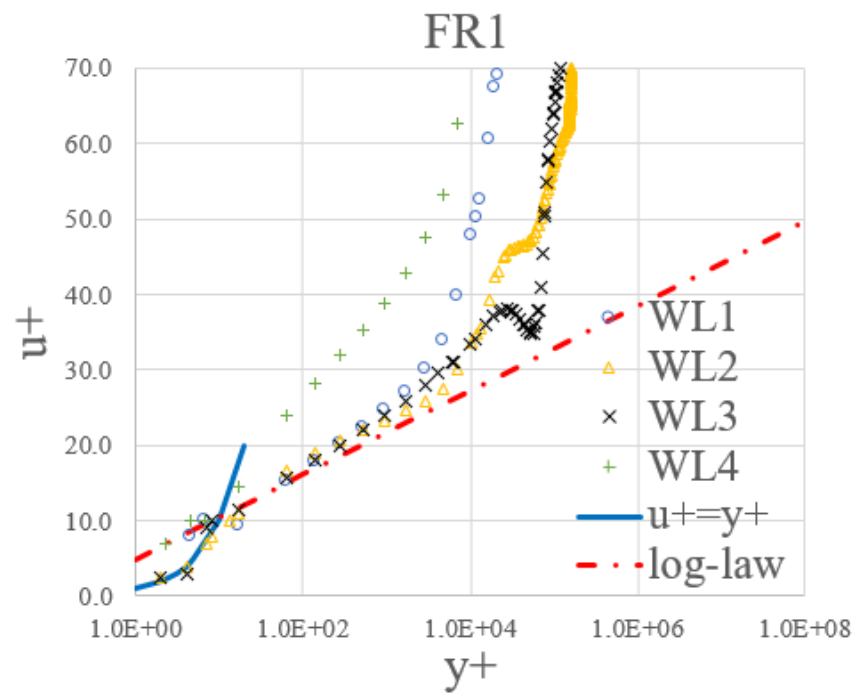

Figure 25 Streamwise $\mathrm{y}_{+} \mathrm{u}_{+}$velocity profile and hull flow separated regions

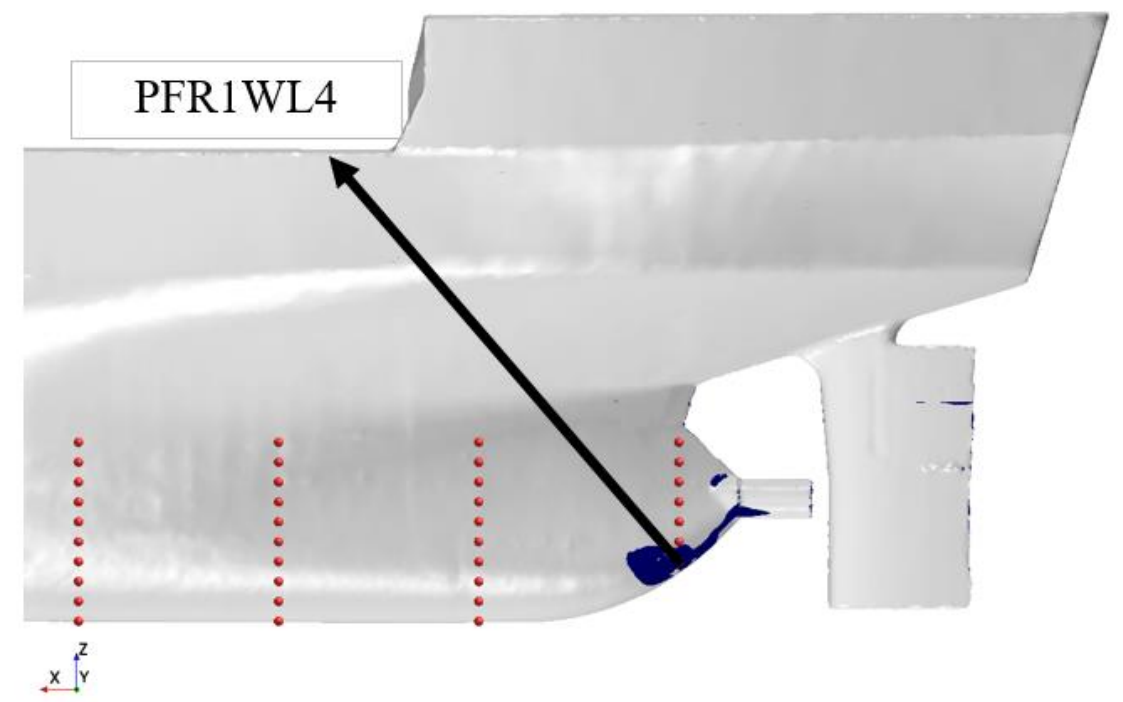

Figure 26 Flow separation on the hull represented by isosurfaces of negative shear stress

\subsection{Vorticity and Vortex Shedding}

Vorticity and vortex shedding from the hull are analysed and also post-processed, with the expectation to find an explanation for the high disturbance of the velocity fields found in the nominal wake field presented in Figure 15. Needless to say, so close to the hub and due to the hull's shape, one may expect to find significant vorticity in the transverse components of the vorticity vectors.

Figure 27 shows the dimensionless vorticity $\left(\frac{\overline{\omega_{l}}}{L} \overrightarrow{U_{\infty}}\right)$ plots in the streamwise $(\mathrm{x})$, spanwise $(\mathrm{y})$ and $\mathrm{z}$ directions, as shown in Figure 15. The vorticity plots confirm that the three components of the 
vorticity are significant in the nominal wake region. However, with three different vorticity components, it is difficult to visualise the actual vortex structures present on the hull.

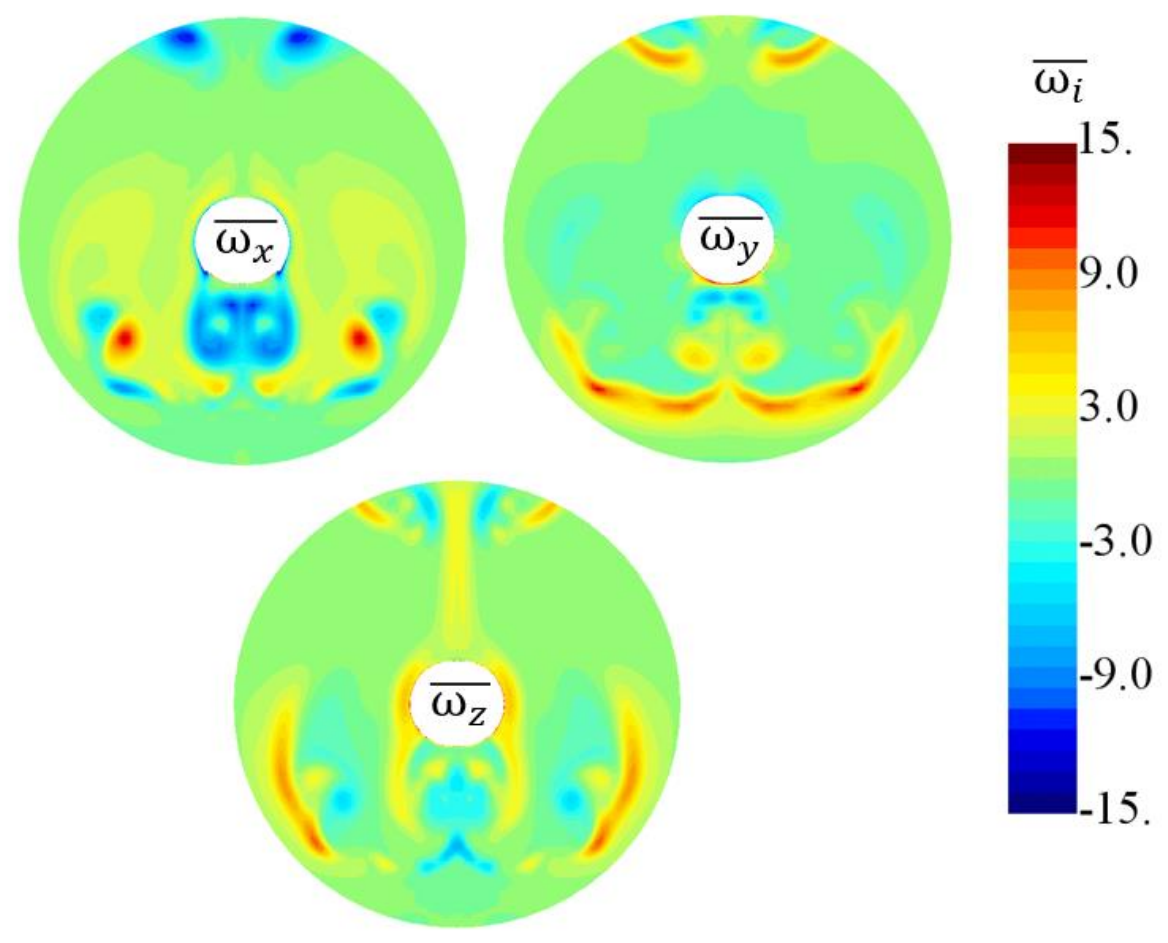

Figure 27 Vorticity fields in $\mathrm{x}, \mathrm{y}$ and $\mathrm{z}$ directions

To visualise the actual vortex structures, an instantaneous snapshot of vortex core locations shed from the hull is shown in Figure 28. These vortex structures are represented using positive isosurfaces of the Q Criterion $Q=\frac{1}{2}\left(\Omega_{i j} \Omega_{i j}-S_{i j} S_{i j}\right)^{1 / 2}$, where $\Omega$ is the rotation tensor, and $\mathrm{S}$ represents the strain tensor (Jeong and Hussain, 1995). In particular, a strong bilge vortex may be not desired as it tends to lower the velocity in the region between the hull and propeller. Such an effect could cause the propeller blade sections to experience a sudden change in the local angle of attack, resulting in sharp changes in local force and cavitation flare-up, hence shaft torque fluctuations and hull vibration from cavitation dynamics.

For Regal, a 3-dimensional representation of the vortex systems served to identify; a strong bilge vortex, two symmetrical side vortex structures and small vertical vortices that are present across the entire hull length. On the one hand, the bilge vortex is present as a vortex sheet which closer to the propeller separates from the hull forming ring-like structures. The ring-like structures were expected, and they appear as a consequence of instabilities of the shear layer arising at the boundary between the slow wake flow passing by the hull and the freestream flow (Doligalski, 2002). This phenomenon has been further confirmed by experiments with blunt bodies (Sakamoto and Haniu, 1990; Dousset and Pothérat, 2010) and it has been reported in towing tank model scale tests (Thornber et al., 2010). 
The bilge vortex is mainly responsible for the slow flow hook-shaped region as seen on the nominal wake field presented in Figure 15. A side vortex is represented as a vortex sheet at mid-girth height on Figure 28. The side vortex sheet is formed due to the change in curvature of the hull and is found to be weak when compared to the bilge vortex. The side and bilge vortex merge downstream forming a single vortex as shown in Figure 21.

With regards to the pattern of the small vertical vortices along the hull surface, we have used the term 'Frame Vortices'. They were suspected to be caused by hull-plate deformation caused at the structural welded frames. It is important to remember that the geometry used during the CFD computations was generated through a 3D laser scan of the ship. This hypothesis was confirmed as the frame spacing was measured, and it was found to be $800 \mathrm{~mm}$, identical to the actual vertical vortices spacing. These vortex structures were fully identified at each hull deformation framing by plotting the $\lambda-2$ criterion (Jeong and Hussain, 1995) at WL3 as shown in Figure 29. This finding is contrary to Kornev et al. investigation (2018), that attributed these vortices to boundary layer instabilities arising at the freesurface. Finally, the vortices shown on the upper region of the propeller disk (Figure 27) are instantaneous structures originating in their closeness to the free-surface.

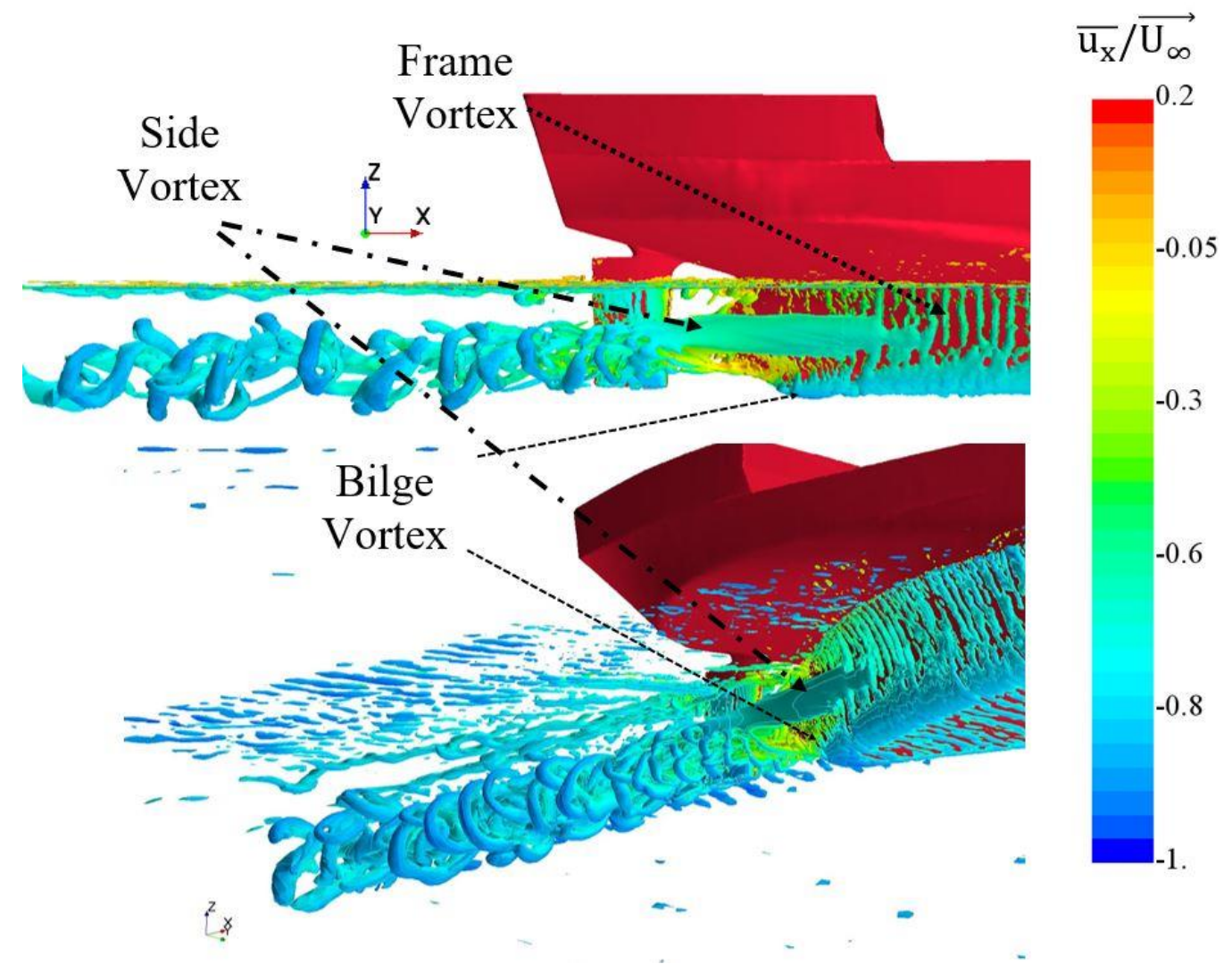

Figure 28 Isosurfaces of Q-criterion showing the existence of different vortex systems 


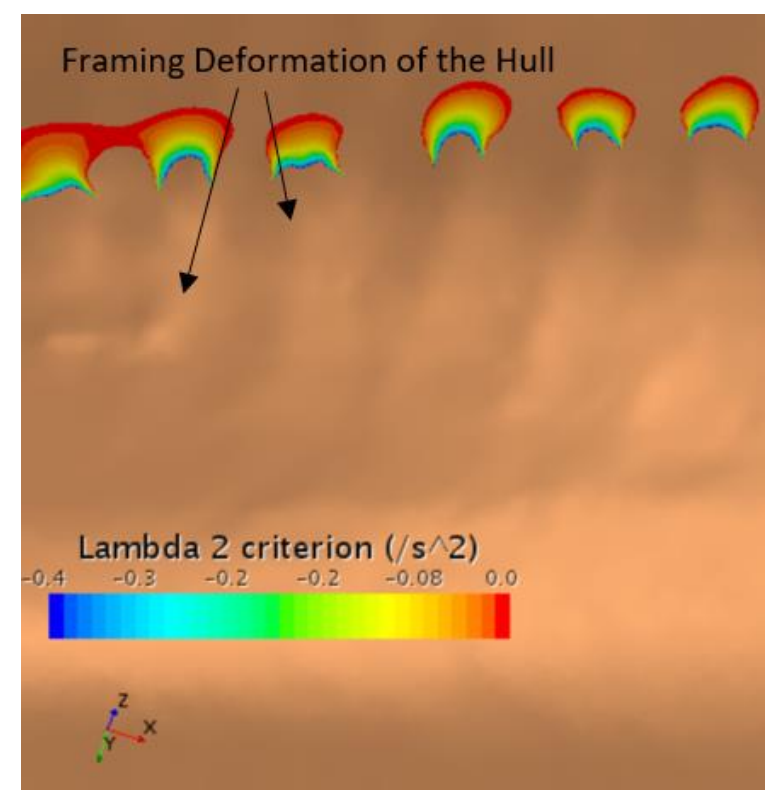

Figure $29 \lambda$ - 2 criterion along WL3

\section{Discussion and Conclusions}

The present research has numerically investigated the ship hydrodynamic performance of a full-scale general cargo ship using extensive flow data on the boundary layer and wake field. The assessment was conducted using an IDDES sophisticated numerical approach able to directly resolve from the Navier-Stokes equations any relatively large turbulent scale vortices contained in the flow. A mesh performance analysis has been demonstrated to be required to verify the performance of the mesh when using a DES approach.

Ship resistance per unit length measurements and pressure coefficient distribution confirmed a pressure resistance coefficient imbalance between the aft and fore end of the ship. This resistance imbalance resulted in an increased pressure drag being the main source of a less than optimum hydrodynamic efficiency for the given geometry.

The hydrodynamic assessment has identified a streamwise pressure gradient along the hull that is initially favourable at the bow and significantly adverse towards the stern of the ship. This negative pressure gradient, together with the bilge vortex create the perfect environment for a local flow recirculation region near the propeller hub. As the flow advances, the near-wall region flow particles retard to a point where it can no longer counteract the pressure gradient, thus separating from the surface. This phenomenon is due to excessive momentum loss near the wall in a boundary layer, that is trying to move downstream against the increasing pressure. The flow separation together with a negative pressure gradient is the leading cause of low-velocity patches seen on the propeller nominal wake region. These regions occupy a large extent of the nominal wake field, therefore causing a substantial detriment on the propeller's performance and the ship hydrodynamic performance. 
The detriment on the ship efficiency is triggered by the loss of momentum that takes place on the aft end boundary layer. Consequently, the boundary layer is too weak to counteract the adverse pressure gradient and together with the effect of the bilge vortex detaches from the hull. This flow detachment contributes to the pressure imbalance between the forward and aft end of the hull, thus increasing the drag of the ship.

In general, the analysis conducted within this work has demonstrated to be essential to a full understanding and explanation of the underlying physical structure of the full-scale aft end flow. In particular, the authors found the present study particularly beneficial when considering key flows for the design of propellers and energy saving devices for full-scale application since it avoids uncertainties from empirical scaling of model scale data and minimises the iterations required to find the optimal ship design. Such a thorough hydrodynamic analysis could avoid the commonly used practice of trial and error that is typically used by some energy saving device designers (Saydam et al., 2018).

\section{Acknowledgement}

The authors would like to acknowledge Giles Thomas for his invaluable contribution to this work. We would also like to recognize Siemens Star CCM+ and Lloyds Register team for their technical support.

\section{References}

Ashton, N., West, A. and Mendonça, F. (2016) 'Flow Dynamics Past a 30P30N three-element airfoil using improved delayed detached-eddy Simulation', AIAA Journal, 54(11), pp. 3657-3666. doi: 10.2514/1.J054521.

Bakica, A. et al. (2020) 'CFD simulation of loadings on circular duct in calm water and waves', Ships and Offshore Structures, pp. 1-13.

Doligalski, T. (2002) 'Vortex Interactions with Walls', Annual Review of Fluid Mechanics, 26(1), pp. 573-616. doi: 10.1146/annurev.fluid.26.1.573.

Dousset, V. and Pothérat, A. (2010) 'Formation mechanism of hairpin vortices in the wake of a truncated square cylinder in a duct', Journal of Fluid Mechanics, 653, pp. 519-536. doi: 10.1017/S002211201000073X.

ISO (2015) ISO 15016:2015 Standard, Ships and marine technology — Guidelines for the assessment of speed and power performance by analysis of speed trial data. Available at: https://www.iso.org/standard/61902.html. 
ITTC (1957) 'ITTC Proceedings of the 8th International Conference', in 8th ITTC 1957. Madrid, Spain.

ITTC (2014a) '27th Propulsion Committee Proceedings', in 27th ITTC 2014.

ITTC (2014b) 'ITTC - Recommended Procedures and Guidelines Preparation and Conduct of Speed / Power Trials 7.5-04-01-01.1', in 27th ITTC 2014.

ITTC (2014c) 'Quality System Manual Recommended Procedures and Guidelines Practical Guidelines for Ship CFD Applications 7.5-03-02-03', in 27th ITTC 2014.

ITTC (2017) 'Uncertainty Analysis in CFD Verification and Validation Methodology and Procedures 7.5-03-01-01', in 28th ITTC 2017.

Jasak, H. et al. (2019) 'CFD validation and grid sensitivity studies of full scale ship self propulsion', International Journal of Naval Architecture and Ocean Engineering, 11(1), pp. 33-43.

Jeong, J. and Hussain, F. (1995) 'On the identification of a vortex', Journal of Fluid Mechanics, 285, pp. 69-94. doi: 10.1017/S0022112095000462.

Kornev, N. and Abbas, N. (2018) 'Vorticity structures and turbulence in the wake of full block ships', J Mar Sci Technol, 23, pp. 567-579.

Larsson, L. et al. (2015) 'Proceedings, Tokyo 2015 Workshop on CFD in Ship Hydrodynamics', Tokyo CFD Workshop.

Larsson, L. and Raven, H. C. (2010) Ship Resistance and Flow, The Principles of Naval Architecture Series.

Lloyds Register (2016) 'Workshop on Ship Scale Hydrodynamic Computer Simulation Proceedings', in. Southampton, UK.

Menter, F. R. (1994) 'Two-equation eddy-viscosity turbulence models for engineering applications', AIAA Journal, 32(8), pp. 1598-1605. doi: 10.2514/3.12149.

Miklosovic, D. S. et al. (2004) 'Leading-edge tubercles delay stall on humpback whale (Megaptera novaeangliae) flippers', Physics of Fluids, 16(5). doi: 10.1063/1.1688341.

Na P. and Moin Y. (1998) 'Direct numerical simulation of a separated turbulent boundary layer', Journal of Fluid Mechanics, 374, pp. 379-405. doi: https://doi.org/10.1017/S002211209800189X.

Patel, V. C. and Sarda, O. P. (1990) 'Mean-flow and turbulence measurements in the boundary layer and wake of a ship double model', Experiments in Fluids, 8(6), pp. 319-335. doi: 
10.1007/BF00217197.

Pena, B., Muk-Pavic, E., Thomas, G. and Fitzsimmons, P. (2020) 'An Approach for the Accurate Investigation of Full-Scale Ship Boundary Layers and Wakes'. doi: https://doi.org/10.31224/osf.io/b86my.

Pena, B., Muk-Pavic, E. and Ponkratov, D. (2019) 'Achieving a high accuracy numerical simulations of the flow around a full scale ship', in Proceedings of the International Conference on Offshore Mechanics and Arctic Engineering - OMAE. doi: 10.1115/OMAE2019-95769.

Ponkratov, D. and Zegos, C. (2015) 'Validation of ship scale CFD self-propulsion simulation by the direct comparison with sea trials results', in Proceedings of the Fourth International Symposium on Marine Propulsors.

Sakamoto, H. and Haniu, H. (1990) 'A study on vortex shedding from spheres in a uniform flow', Journal of Fluids Engineering, Transactions of the ASME, 112(4), pp. 386-392. doi: 10.1115/1.2909415.

Saydam, A. Z., Gokcay, S. and Insel, M. (2018) 'CFD based vortex generator design and full-scale testing for wake non-uniformity reduction', Ocean Engineering, 153, pp. 282-296. doi: 10.1016/j.oceaneng.2018.01.097.

Schlichting, H. et al. (2017) Fundamentals of Boundary-Layer Theory, Boundary-Layer Theory. doi: 10.1007/978-3-662-52919-5_2.

Shur, M. L. et al. (2008a) 'A hybrid RANS-LES approach with delayed-DES and wall-modelled LES capabilities', International Journal of Heat and Fluid Flow, 29(6), pp. 1638-1649. doi: 10.1016/j.ijheatfluidflow.2008.07.001.

Shur, M. L. et al. (2008b) 'A hybrid RANS-LES approach with delayed-DES and wall-modelled LES capabilities', International Journal of Heat and Fluid Flow, 29(6), pp. 1638-1649. doi: 10.1016/j.ijheatfluidflow.2008.07.001.

Spalart, P. R. et al. (1997) 'Comments on the feasibility of LES for wings and on a hybrid RANS/LES approach', Advances in DNS/LES, 1(JANUARY), pp. 4-8.

Spalart, P. R. et al. (2006) 'A new version of detached-eddy simulation, resistant to ambiguous grid densities', Theoretical and Computational Fluid Dynamics, 20(3), pp. 181-195. doi: 10.1007/s00162006-0015-0.

Thornber, B., Starr, M. and Drikakis, D. (2010) 'Implicit large eddy simulation of ship airwakes', Aeronautical Journal, 114(1162), pp. 715-736. doi: 10.1017/S0001924000004218. 
White, F. M. (2011) Fluid Mechanichs, 7th Edition, McGraw-Hill,New York. doi: 10.1016/B978-012-546801-5.50017-7.

Wilcox, D. C. (1993) Turbulence modeling for CFD, Aiaa. doi: 0963605151.

Xing, T., Carrica, P. and Stern, F. (2010) 'Large-Scale Rans and Ddes Computations of Kvlcc2 At

Drift Angle 0 Degree', (3), pp. 2-7. 